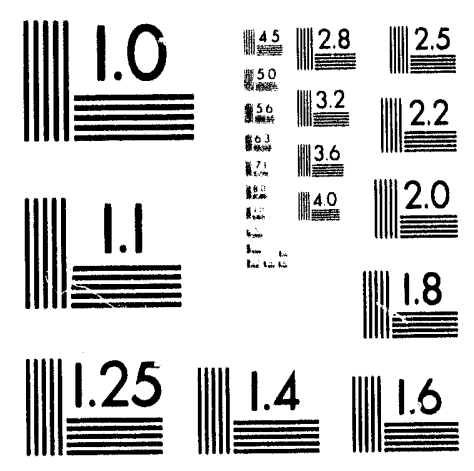



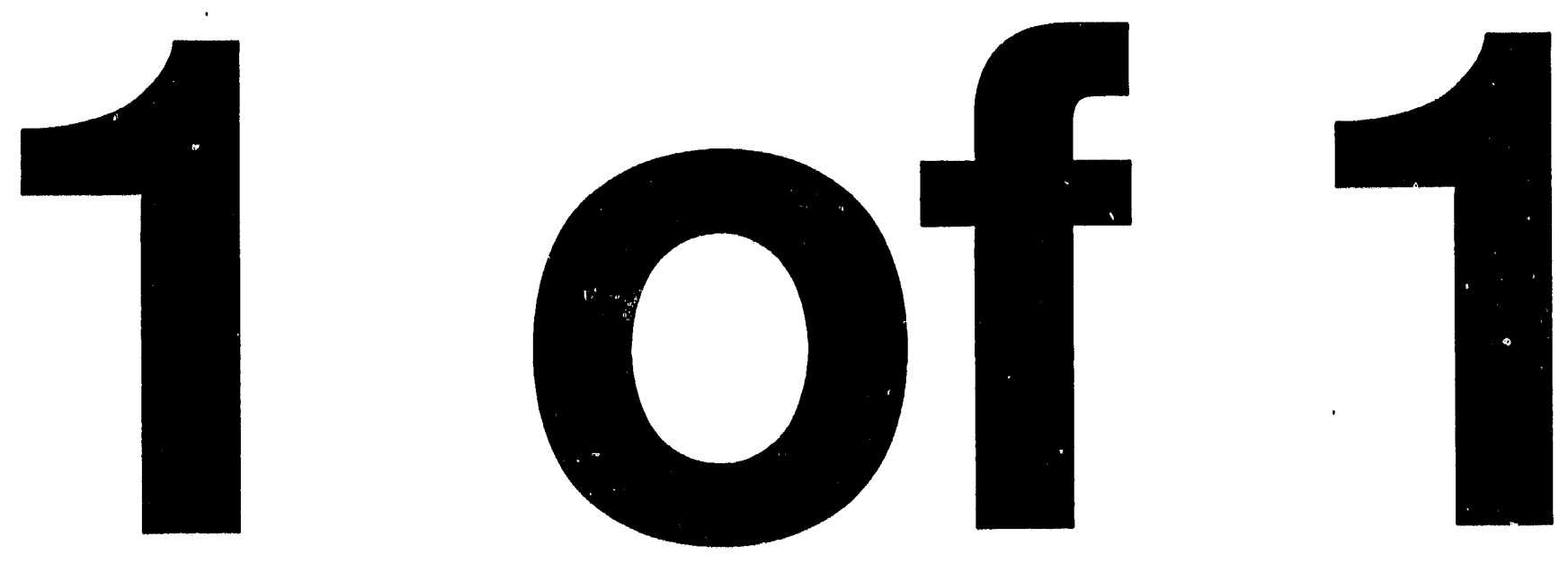


\title{
Center for X-Ray Optics
}

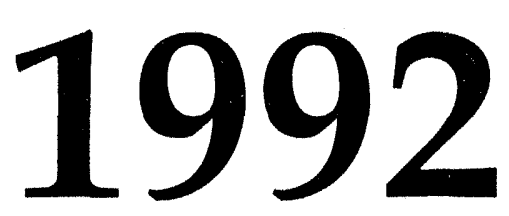

\author{
Materials Science Division \\ Lawrence Berkeley Laboratory \\ University of California \\ Berkeley, California 94720
}

LBL-34462

Printed August 1993

This work was supported by the Director, Office of Energy Research, Office of Basic Energy Sciences, Materials Science Division, of the U.S. Department of Energy under Contract No. DE-AC 103-76SF00098, by the U.S. Department of Defense, Air Force Office of Scientific Research under Contract No. F49620-87-K-(0)01, and by the Advanced Research Projects Agency under ARI'A Order AO-8335. 


\section{Contents}

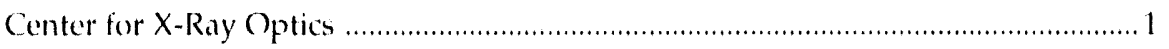

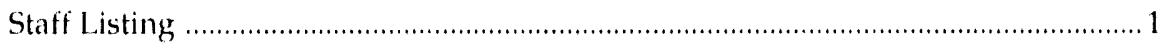

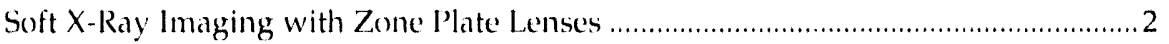

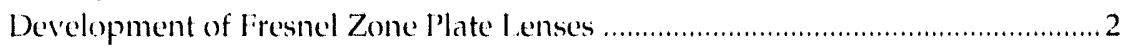

Soft X-Ray Microinterferometer for Materials Studies ........................................ 3

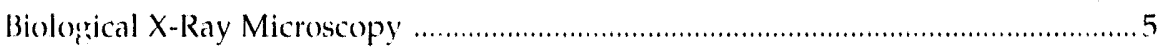

Extreme Ultraviolet Lithography for Nanoelectronic Pattern Transfer ...................... 7

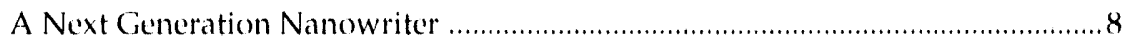

Reflective Masks for Extreme Ultraviolet Lithography ....................................9

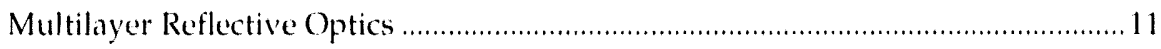

Studies of Roughness and Diffuse Scattering from Mirrors .............................. 11

Controlling Multilayer Period Variation for Imaging Applications .................... 13

Tarnishing and Restoration of Mo/Si Multilayer X-Ray Mirrors........................ 14

Electron Beam Microanalysis of Light Elements ............................................... 15

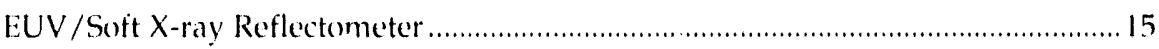

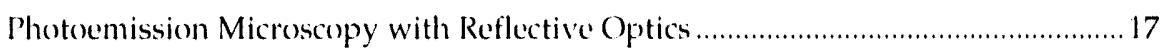

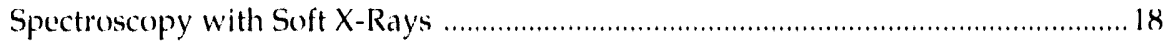

Monochromators and Spectrometers .......................................................... 19

Spatially and Temporally Coherent Beamlines ..................................................19

High Resolution VUV Monochromator for the Chemical Dynamics Beamline . 20)

High Resolution Monochromator for Carbon Related Studies ............................21

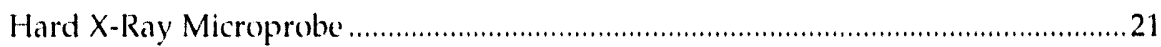

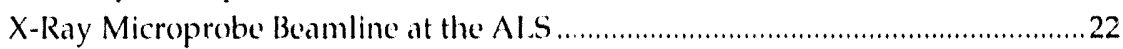

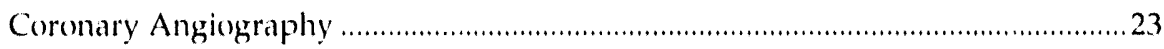

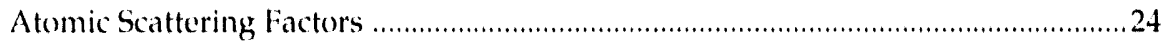

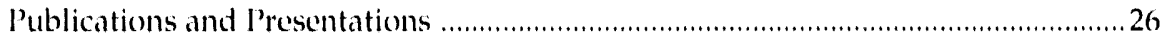




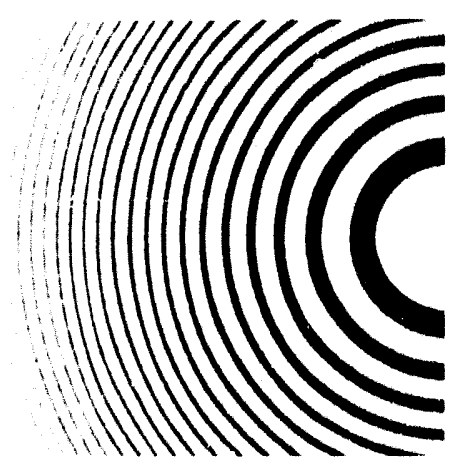

\section{CENTER FOR X-RAY OPTICS}

I

In 1092 the Center for X-Ray Optics (CXRO) continued its two complementary roles: demonstrating the capabilities and uscefulness of the $x$-ray and ultraviolet regions of the spectrum and developing equipment and techniques to make those capabilities widely and readily available. Efforts continue to develop state of the art $x$-ray lenses and mirrors, monochromators optimized for high resolution and throughput, optical systems for the utilization of partially coherent radiation, and applications across the physical and life sciences.

High-resolution $x$-ray microscopy continues to be a prominent activity. Soft-x-ray microscopy based on fresnel zone-plate lenses has provided images of features as small as $300 \mathrm{~A}$ in experiments at the Berlin Electron Synchrotron (BESSY). Spatially resolved studies of materials have been conducted with colleagues at both Wioconsin and Brookhaven. Biomicroscopy studies have been explored with colleagues at Göttingen and Stony Brook. In the hard-x-ray regime, a microprobe, based on multilayer-coated reflective optics, has achieved $2-\mu \mathrm{m}$ spatial resolution at the National Synchrotron Light Source (NSLS) and has been used in a large number of applications in the life and physical sciences. The microprobe is presently in use at ESRF in Grenoble, and will soon be used among the first experiments at LBL's newly commissioned Advanced Light Source (ALS).

Scientific and Technical Staff

1) Altwind thichiri

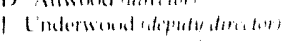

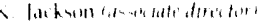

1. Anderom

1. Hathut

1. Heneyili

I. Busker

h. (h.1pm.n!

R. I Hallow

1. Ironham

G.ullih som

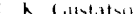

Henherisenth

II $1 \mathrm{lu}$

1) kiming

S klunghin

II kiske

I. hintright

II. I 1 ,

II Velenk

II Nextr-llat

1 Nothat

I switll

$k$ lakitherrs

1. Hounpun

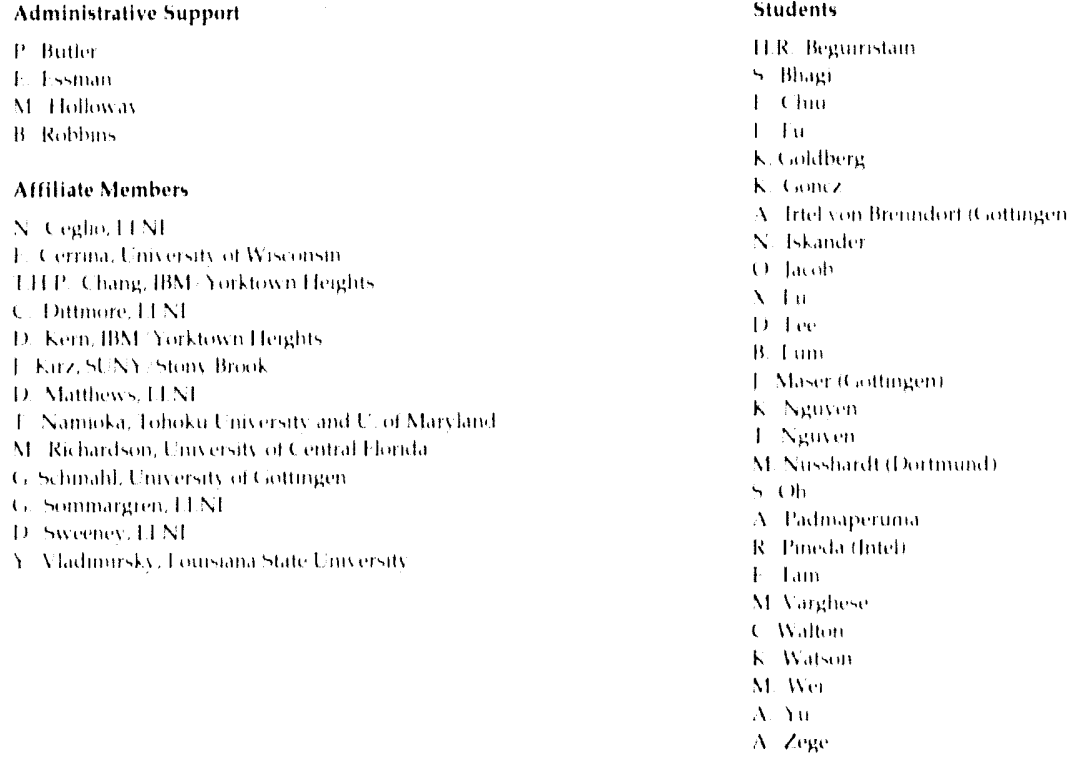

Administrative Support

I) Butlier

1. 1 suman!

II Hollewis

B Rohbum

Affiliate Nembers

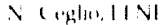

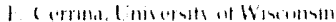

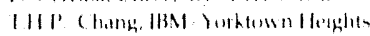

(. Bttmore, $1.1 \mathrm{NI}$

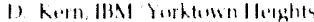

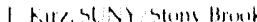

1) Vhathews $|1, \mathrm{~N}|$

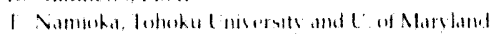

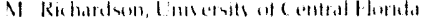

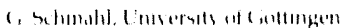

1. Gummarghen, $11 \mathrm{~N}$

1) bereme. 11 N1

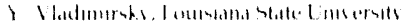


In the long-term effort to develop high-reflectivity mullilayer contings for extreme-ultraviolet and soft-x-ray optical elements, such as mirrors and gratings, we continued investigating the structure and stability of various multilayer pairs and developed a new, highly versatile reflectometer based on a laser-plasma $x$-ray source and a high-throughput monochromator.

A new program in projection lithography has been initiated whose goal is to provide critical metrologies and optical test capabilities for a national program in nanoelectronic pattern transfer. This joint initiative brings researchers from CXRO and the University of California at Berkeley together with representatives of the semiconductor industry. The goal is to further the use of extreme ultraviolet (EUV) radiation in the fabrication of computer chips with feature sizes of order $0.1 \mu \mathrm{m}$. The photon beams from the ALS are well suited to this research, as is our experience with high resolution electron beams and nanometer-scaled metrologies for fine pattern writing.

With completion of the ALS anticipated in 1993, a number of beamline and endstation projects are underway there, including a high resolution zone plate microscope, "at wavelength" $(130)$ A) interferometry of reflective optics for nanoelectronic pattern transfer, a metrology beamline for spatially and spectrally resolved absolute spectrometry, and a hard $x$-ray microprobe as mentioned above. A new high resolution electron beam "nanowriter" for diffractive optics, mask writing and materials research is also under development.

Extending high-resolution visible-light and ultraviolet imaging techniques into the soft-x-ray region of the spectrum offers several special advantages. The relatively short wavelengths, ranging from several angstroms to perhaps 100 angstroms, permit researchers to both "see" and "write" smaller patterns. Furthermore, the associated photon energies, ranging from approximately 100 to several thousand electron volts (eV), span the primary resonances of many elements. Resonances constitute a sensitive mechanism for element identification, for elemental mapping, and, in some cases, for determination of chemical bonding. Working with collaborators worldwide we helped to advance the technology of soft-x-ray imaging and continued to demonstrate potential applications in both the physical and life sciences. Features as small as $300 \mathrm{~A}$ may be seen in our best images.

In microscopy with soft $x$-rays, the key optical component that ultimately determines performance is the objective lens. Ordinary refractive lenses like those used for visible light, which transform the phase of a wavefront without changing the amplitude, cannot be used at $x$-ray wavelengths because available materials do not give enough phase shift and are not sufficiently transparent. To date the best resolution obtained in this spectral region has been obtained with Fresnel zone plate lenses. Zone plates are thus the lenses of choice for the highest spatial resolution, particularly for energies greater than $200 \mathrm{eV}$.

In pressing toward the fundamental diffraction limit of lens performance, accurate placement of the zones (alternate bands of transmissive and opaque material) is important. The maximum placement error should be less than a fraction of the smallest zone width of the lens. This is a

\section{Soft-X-Ray Imaging with Zone-Plate Lenses}

\author{
Development of \\ Fresnel Zone-Plate \\ Lenses
}


Figure 1. This fresnel zone-plate leils, made of nickel, is representative of our slate-of-llheart accomplishments. It has a smallest zone width of $350 \mathrm{~A}$. (a) shows the central zones and (b) shows the fine structure of some of the smaller anes.
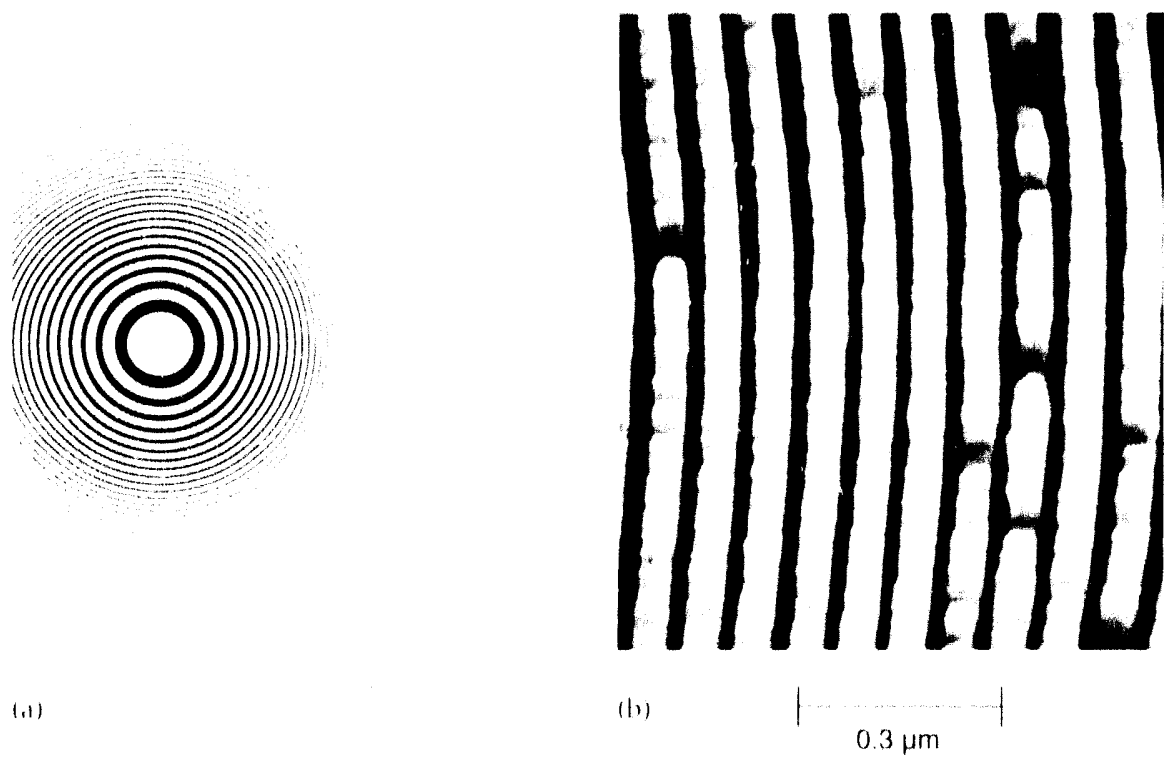

X[33 9375,004

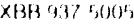

formidable challenge, since our highest-resolution lensen, like the one shown in ligure 1, hawe ane widths of order $350 \mathrm{~A}$. Achicement of the repuired accuracy at these dimensions, especially across large (50)-pmdiametere lenses, in at the frontier of metrolegy and microfabrication. In collaboration with researchers from the Unicersity of Giettingen, we have been using and characterizing the high resolution zone plates in the (ioittingen $x$-ray micrescope t the Berlin Iilectron Synchrotron (BLSSY). Although measurements of the micrescope's optical performance indicate that the diffraction limit has not been reached, images of test patterms show that features as small as $3(0) \AA$ are visible. Our newest nickel zone plate lense's have achieved both high spatial resolution and high diffractive efficiency.

The accurate measurement of the $x$-ray optical properties of materials,

Soft X-Ray Microinterferometer for Materials Studies both attenuation and phase shift, is technologically and scientifically important. A new type of interferometer has been designed and build to make direct measurements of the phase shift as well an attenuation through a test sample on a sub-(). $1 \mu \mathrm{m}$ spatial scale. This interforometer uses all diffractive optics: two gratings and a rone plate, in a configuration similar to an imaging $x$-ray microscope. Figure 2 shows the physical set up. A grating with a period of $\Lambda=0.4 \mu \mathrm{m}$ is imaged by a zonce plane lens $(D)=100 \mu \mathrm{m}, \Delta r=0.06 \mu \mathrm{m})$. In the zone plate back focal plane the diffracted order of the grating form a series of spots for the ()th, $+/-1,+/-2, \ldots$ diffracted orders. An aperture blocks all orders except the +1 and -1 . At the image plane these two orders recombine to form an image of the original grating, which has half the magnified period since the zero order is missing. Because the peried of this interference pattern is smaller than the resolution of the micro-channel plate detector (MCI), a final grating is used to form a Moirc pattern that is observable on the MCP. Figure 3 shows the sub-micron resolution flexure stages used to position the zone plate, aperture, and sample each in $X, Y$, and $Z$. If the sample under test is 
Soft X-ray Micro-Interferometer

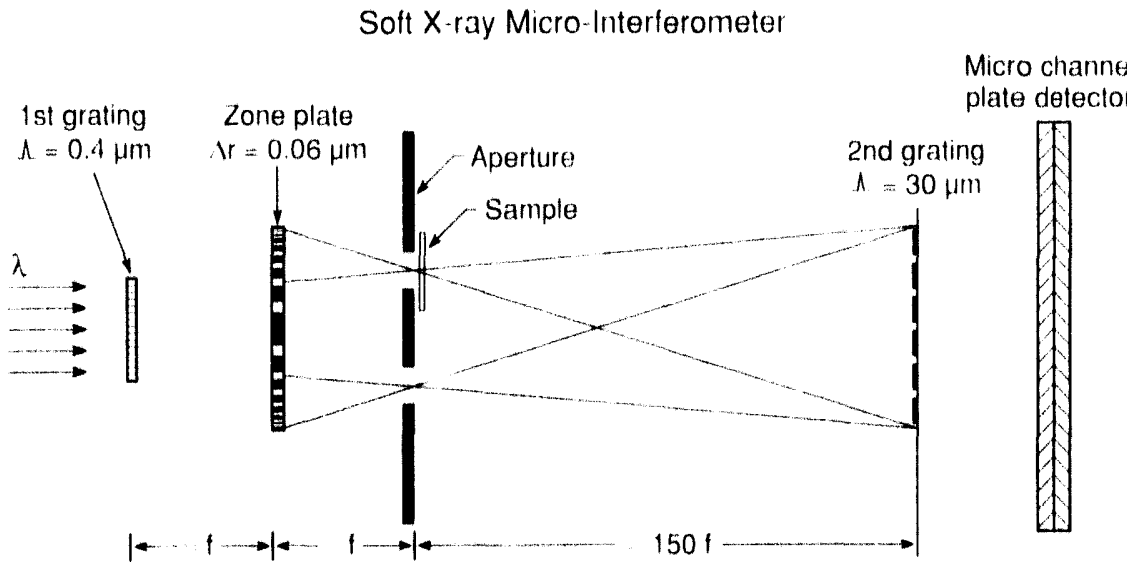

XE11 937.4822

inserted in only one of the diffracted orders (which are 30 mom apart), the amplitude (i.e., contrast) and relative phase of the observed Meire fringes spreat out over the entire MCl'area. A sine poltern is measured by trinslating the final grating through one or more periods while recording the $x$-ray count rate as a function of position. Proliminary experimental results at the NSil $S$ XI beamline demonstrate that the interferometer works and that fringe patterns have been obtained. Further effort is needed to accurately measure the optical constants of materials on a sub-(1). 1 Hm scale.

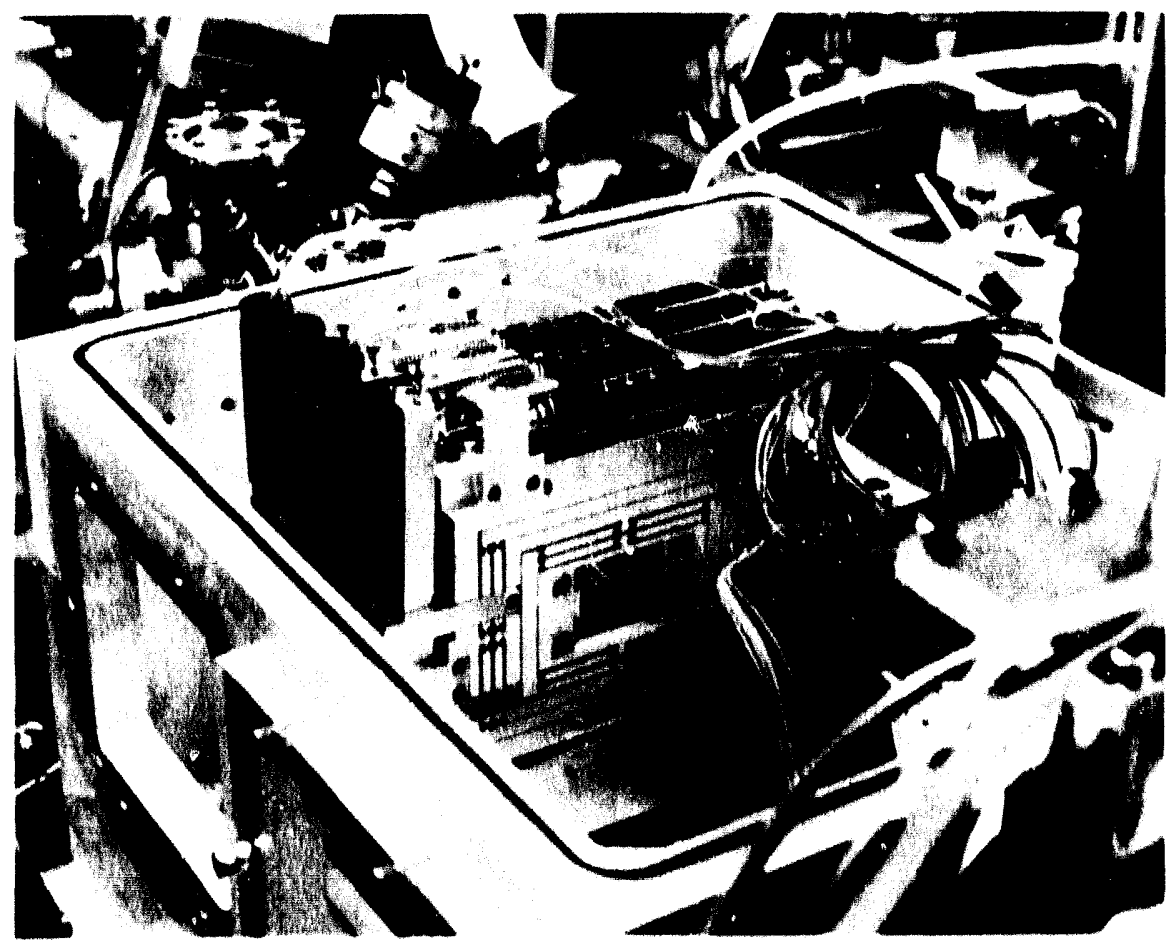

(2)36 $03 / 4854$ ligure 2. Schemalic layout of the soft r-bay microinterferomeder. ligure 3, Soll x-ray microinterforomeder housing with zone plate, aperalure, and sample stages. 


\section{Biological X-Ray Microscopy}

Compared to visible light microscopy, $x$-ray microscopy has a greatly improved resolution. The key optical elements in achieving this resolution are the zonceplate lenses described above. Although $x$-ray microscopy does not compete with electron microscopy in terms of resolution, it offers unique advantages, indeluding the ability to image thick $(1-10 \mu \mathrm{m})$ intact samples, and to do this in an aqueous physiologically relevent environment. The methed is based on the interaction of $x$-rays with matter in the warelength range from 22-44 $\AA$ (the so-called "water window"). In this range, water absorbs relatively weakly, allowing contrast to be provided by naturally occurring coll components such as proteins, nucleic acids, and carbohydrates. Because of the penetrating power of $x$-rays, information can be obtained from thick samples that are not accessible to other techniques, including high voltage clectron, atomic force, and near-field microscopies. The x-ray microscope can also be used as a high resolution analytical tool. In conjunction with a tunable synchrotron-based $x$-ray source, the specific absorption properties of different elements can be used to obtain elemental maps and other chemical information from the simples.

$X$-ray microscopes can be either conventionally designed imaging $x-$ ray microscopes or scanning $x$-ray microscopes. The most elaborate microscopes of either type are the Cïttingen imaging $x$-ray microseupe at BESSY in Berlin and the scanning $x$-ray microscope at the NSIS, Brookhaven National Laboratory. The two microscopes serve complementary needs. Using an uncomplicated design, the imaging microscope provides images with the highest resolution combined with the shortest expesure times. The scanning microscope offers a distinct adrantage of high spatial resolutiom images with minimal radiation dose. We have conducted experiments at both facilities during the past year and oonducted a design effort to build both microscope types at the Advanced Light Source.

The high resolution of $x$-ray microscopy is achieved with zone plate lenses, which we build with an over all efficiency of $7.3^{\prime \prime}$ and an outermost zone width of 350 () $\AA$-cleser to the theoretical limit than any other zome plate with this resolution. These ane plates are regularly used with the scanning electron microscope at Brookhaven, and the highest resolution experiments to date have been performed with these zone plates on the imaging $x$-ray microscope at BESSY. The high efficiency of these zone plates enables reduction of the radiation dose te a biological sample, while a further reduction is achieved by using a highly afficient detector. To utilize these advantages, we installed a special electronic camera on the BESSY microscope using a back-illuminated thimmed $C(D)$ as a recording device, which achieves a $62 "$ " efficiency at the most used $x$-ray wavelength of $24 \AA$. This camera reduces both the expesture time and the radiation applied to the sample, each by more than an order of magnitude.

Our experiments at the existing $x$-ray microscopes have addressed two needs: first was evaluation of the performance of such critical parts of $x$ " ray microscopes as zone plates and detectors. Second was enhancement of ou:r knowledge of preparation techniques, contrast mechanisms, and related topics. Fxamples of biological samples imaged using the instruments at BESSY and Brookhaven National Laboratory are shown in Figures 4 and 5. 
The Center for X-Ray Optics has begun to design complementary imaging and scanning $x$-ray microscopes for the Advanced Light Source to form a Biological X-Ray Microscopy Resource Center. Because the ALS is the brightest source of tunable $x$-rays available worldwide, these microscopes will combine the highest possible resolution with shortest possible exposure times. The first microscope at the AlS will be an imaging type using bending magnet radiation, to be available in 1994.

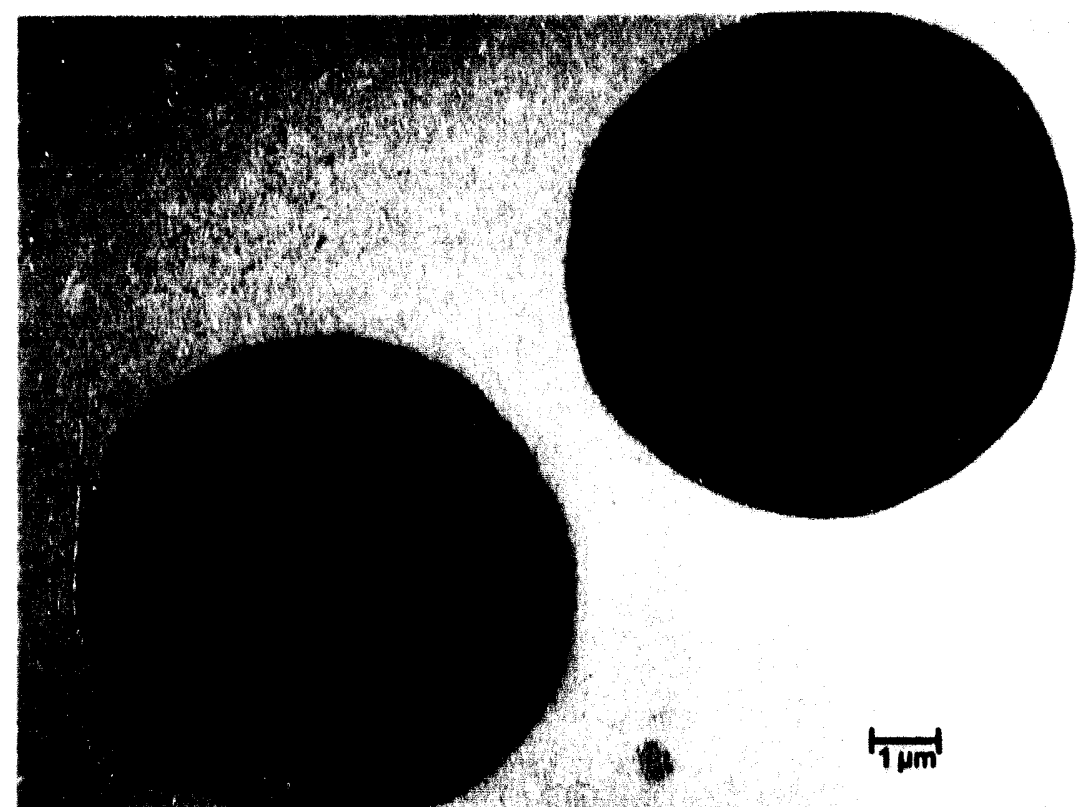

ABC 937.4855

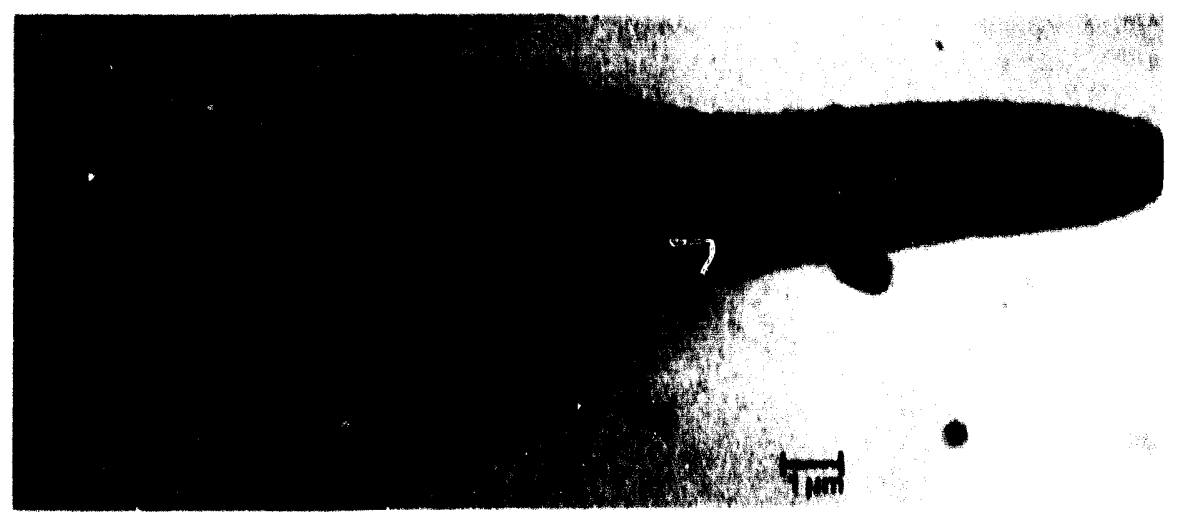

$\times B 3937.500 \%$
Figure 4. Intact human red blood cells, one infected by malaria parasites, both imaged with soft $x$-rays. These studies indicate that $x$-ray microscopes are well suited to studying the development of the parasile within the cell, and may provide useful information regarding the pathology of the disease. These images are part of a study by Drs. C. Magowan and M. Moronne (I.BL). The image was taken with the Gibltingen $x$-ray microscope at BESSY in Berlin.
Figure 5. Spermhead of a marsupial mouse (Sminthopsis) taken with the Goittingen x-ray microscope at BESSY using the new back-illuminated CCD camera and a high efficlency nickel zone plate $1350 \mathrm{~A}$ outermost eone width). this image Is part of a study by Drs. William Breed (U. of Adelaide) and Rodney Balhorn (L.L.NI.). 


\section{Extreme Ultraviolet Lithography for Nanoelectronic Pattern Transfer}

Figure 6. Spallally and temporally filtered radiation at the ALS will be used for "at. wavelength" Interferometric lesting of optics. The beamline design is shown in ligure 19.
Since the creation of the first integrated circuit in 1960 there has been an ever increasing density of devices manufactured on semiconductor substrates. The very large scale integration (VSLI) era from the mid 1970)s to the present has seen chip densities from $10(0),(0)(0)$ transistors per chip to over 1 million per chip. This increasing device count was accomplished by a shrinking minimum feature size that includes linewidth, spacing, and contact dimensions, from $2 \mu \mathrm{m}$ in the late 1970) to less than $0.75 \mu \mathrm{m}$ in current 4-megabit dynamic random access memories (DRAMs). The challenge to continued U.S. industrial competitiveness in microelectronics will be development of new techniques of lithography and pattern transfer at minimum fenture sizes of $0.1 \mu \mathrm{m}$ and smaller. The lithography program at the Center for X-Ray Optics focuses on the enabling technologies that are essential for EUV optical imaging systems; Imaging systems that will be required for $0.1-\mu \mathrm{m}$ features and 1-gigabit integrated circuits by the year 20(0). The program concentrates on the develepment of EUV interferometry for testing optics at a wavelength of $1.30 \AA$, nanofabrication facilities for diffractive optics and reflective masks, high placement accuracy over large areas, and related research activities in optics, device physics, pattern transfer, and requisite metrologies.

New facilities will include bending magnet and undulator beamlines at the Advanced Light Source. The undulator beamline will have a unique EUV interferometer for "at wavelength" characterization of high numerical aperture reflective optics based on the use of partially coherent undulator radiation. A bending magnet beamline at the AlS will be fitted with both EUV and soft $x$-rany metrology stations for spatially and spectrally resolved absolute spectrometry, including precise messurements of mirror reflectivity, resist sensitivity, uptical efficiency, refractive index, and other quantities.

In addition, a manofabrication facility, described below, with next generation direct-write olectron-beam capability (a "nanowriter") for making best-in-the-world diffractive $x$-ray optics, reflective mask patterns, and other structures is being built.

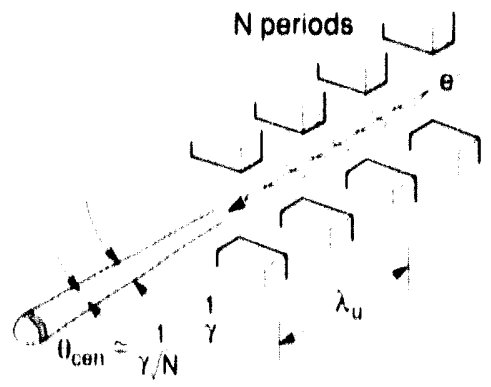

$$
\begin{aligned}
& \lambda_{x}=\frac{\lambda_{u}}{2 \gamma^{2}}\left(1+\frac{k^{\prime}}{2}+\gamma^{2}()^{2}\right) \\
& \text { in the central radiation cone: } \\
& \lambda=N \text { and } \theta_{c e n}=\frac{1}{\Delta \lambda}=N / N
\end{aligned}
$$

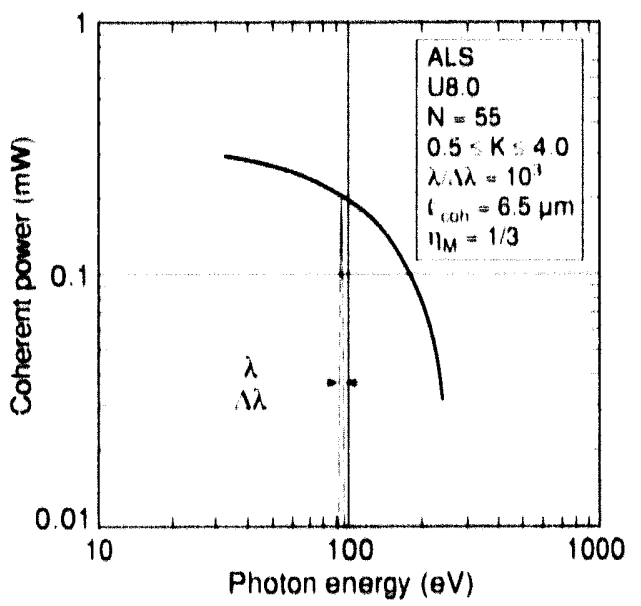




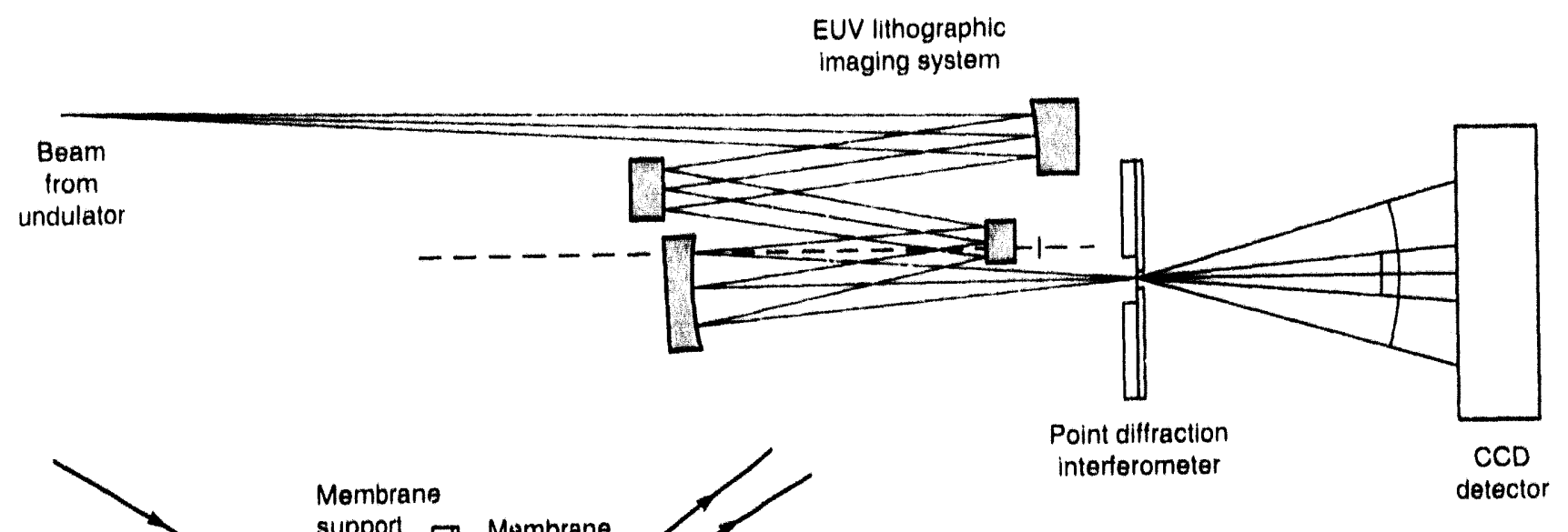

For fabrication of state of the art diffractive optics (\%one plates and gratings), olectron-beam nanolithography is a key technology. To overcome limitations with commercially available electron-beam machines, LBL has taken on an ambitious project to develop a next-generation "nanowriter." The nanowriter and other instrumentation will allow research in the areas of high resolution diffractive optics, nanoelectronic patterning, materials processing, studies of quantum transport device physics, and the development of requisite metrologies for the overlay of $0.1-\mu \mathrm{m}$ patterns across square centimeter areas in future nanoelectronic devices. Our goal is to build a working machine that is superior to existing systems in terms of resolution, placement accuracy, and throughput. Some of the aggressive specifications to be met are a $2.5-\mathrm{nm}$ spot size within a 100 - $\mu \mathrm{m}$ diameter field size, less than 25- $\mathrm{nm}$ stitching error between fields over square centimeter dimensions, and up to 10(0)-MHz beam stepping rate with arbitrary shape exposure.

During the development, great emphasis is given to keeping the system flexible, expandable, and tumable to meet different requirements for placement accuracy, minimum feature size, and moderate throughput. Of the 14 subsystems identified for the nanowriter, three represent a special challenge in that their specifications reach beyond what has been done to date. Two of them, the integrated final lens and deflection system, and the ultra-high precision specimen stage, will be co-developed by CXRO

\section{A Next-Generation Nanowriter}

Figure 7. Point differaction interfe ometry will be used for testing optical components such as lenses, mirrors, and beam splitters, as well as full imaging systems. This work is done in collaboration with Gary Sommargren of LLNL. 
Figure 8. Schematic of the "nanowriter" electron-beam system under developinent by LBL and industrial partners.
Reflective Masks for Extreme Ultraviolet Lithography

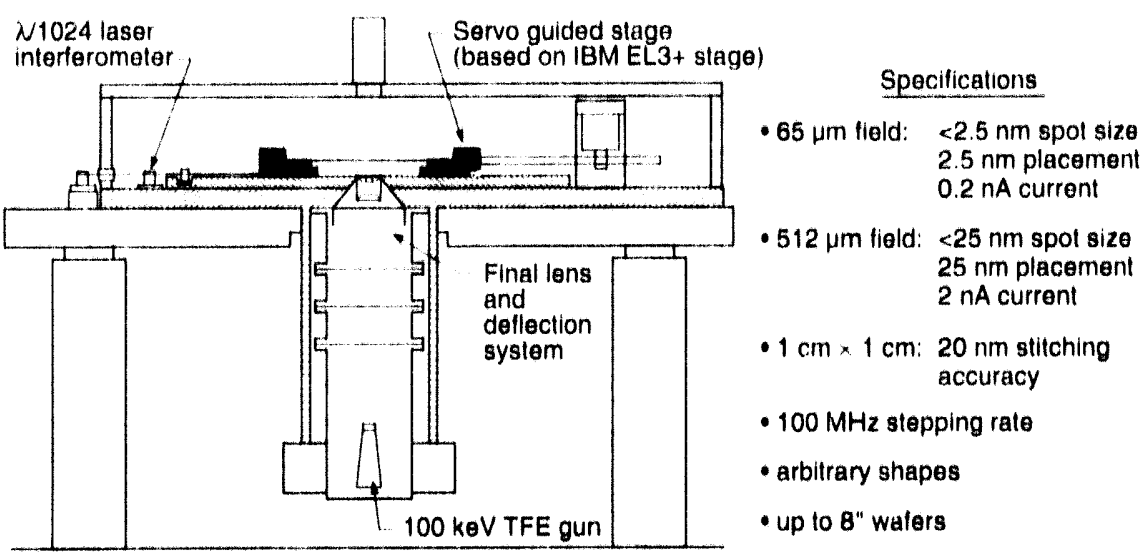

XBL. 9212.5863

scientists and an outside vendor. The third, the high-speed digital pattern generator, is currently under development by CXRO.

Other subsystems, including job preparation software and proximity effect correction, as well as the overall tool control hardware and software, are also being developed within CXRO, which will also be responsible for overall project management and system integration. Negotiations have been started with IBM Research, where prototypes of the tool control system and portions of the proximity correction are being used to control several nanolithography electron-beam machines. The nanowriter is expected to be operational in 1996 .

The development of reflective masks is a critical component of the emerging program in extreme ultraviolet lithography. A reflective mask is made by patterning an absorber layer above a multilayer reflective coating. To be imaged with fidelity onto the wafer, all factors that affect its imaging characteristic must be well understood. The abrupt topography of the absorber layer and the distributed reflection from the coating make calculations of the reflected image complex. As a result, electromagnetic simulations using time-domain finite-difference techniques are used to study the effects of topography and multilayer coating defects on the reflected areal image.

In early investigations we find that the illumination angle should be of no more than 10 degrees off-normal incidence and that only a thin absorber layer, approximately $100 \mathrm{~nm}$, of germanium or carbon is needed for good image contrast. With near normal-incidence illumination and a thin absorber layer, the absorber overlayer reflective mask is relatively insensitive to variations in incidence angle in absorber profile, making it a robust mask geometry.

The effect of multilayer defects on the reflected image, however, can be dramatic. For instance, a substrate defect of one tenth the resolution limit can produce a $70 \%$ reduction in image intensity. Experimental studies are underway to confirm these predictions. In addition to pattern transfer studies, high resolution transmission electron microscopy and scanning tunneling microscopy are being used to study the propagation of a substrate defect through the multilayer stack and its effect on the multilayer structure. 


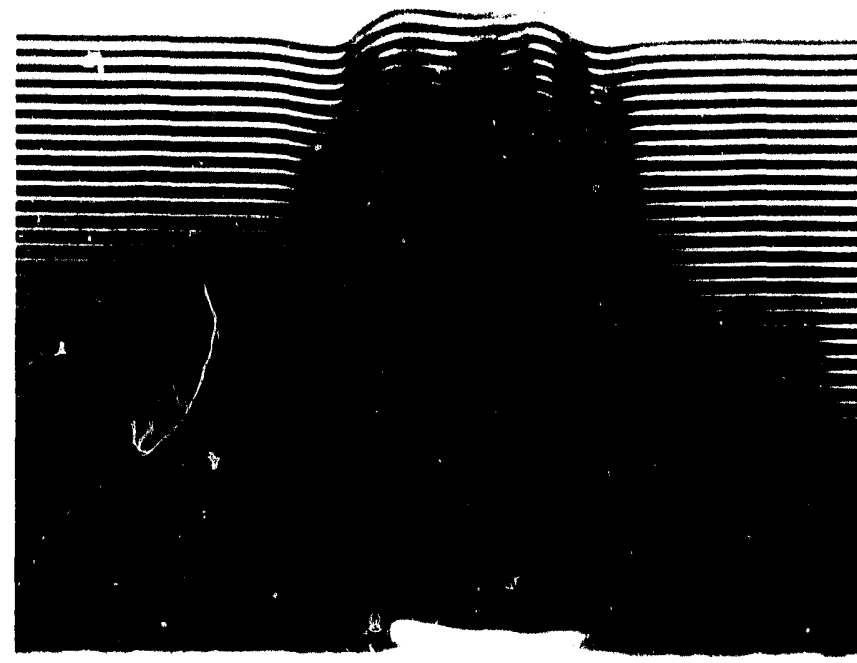

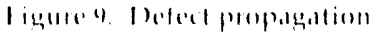

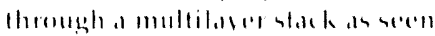

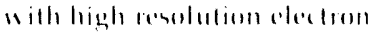
micruscops.

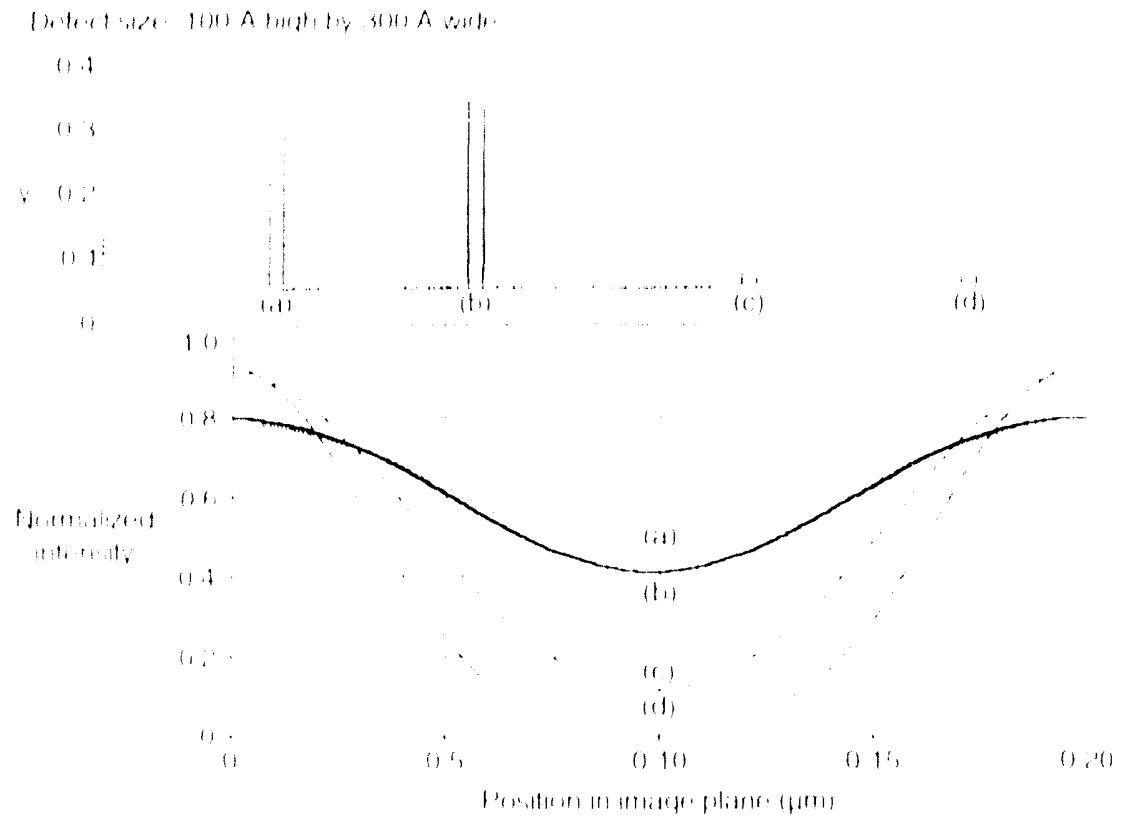

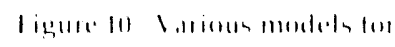

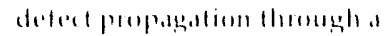
multilasur monk (lop), and

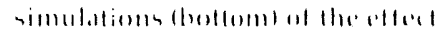
and arral imstge intromils. 


\section{Multilayer Reflective Optics}

Studies of Roughness and Diffuse Scattering from Mirrors
Multilayer coatings are effective reflectors of $x$-rays over a broad wavelength range. The wavelengths and angles of incidence for which they are highly reflective are determined by the Bragg Equation with the $\mathrm{d}$ spacing equal to the period of the multilayer; that is, the sum of the thicknesses of one high- $Z$ and one low- $Z$ layer. Our effort encompasses fabricating multilayers via sputtering techniques, advancing the applications of multilayers in a variety of forefront experiments, and conducting fundamental research into multilayers themselves to improve them and elucidate their performance limits.

In the U.S and abroad, multilayers fabricated in our laboratory have been incorporated into a wide variety of $x$-ray optical systems at photon energies ranging from the extreme untraviolet (EUV) to the hard $x$-ray regions of the spectrum. In the EUV and soft $x$-ray regions multilayer applications include simple dispersing elements for spectroscopy, normal incidence imaging optics of interest, e.g., in projection lithography, polarization controlling and converting devices, and coatings on gratings to extend their high energy range. In the hard $x$-ray region applications typically take advantage of the broad bandwidth of multilayers compared to natural crystals, and include broadband dispersing elements, power filtering mirrors, and coatings for microfocusing mirrors.

Interface roughness reduces specular reflectance and produces diffuse scattering, both of which are critically important in specifying the performance of $x$-ray mirrors. These effects are especially severe for multilayer mirrors because they operate at large scattering vector or momentum transfer compared to total reflection mirrors, where they are most pronounced. We study the roughness of x-ray mirrors by measuring the specular reflectance and diffuse scattering from a variety of samples, including flat polished substrates, sputtered single films, and multilayers. The surfaces are also measured by optical interferometry and atomic force microscopy to compare the roughness values obtained from these different techniques. Microstructural characterization using TEM and high-angle $x-$ ray scattering are applied to multilayers and films in some cases. In addition to providing fundamental data on $x$-ray mirror performance, these measurements allow us to test theoretical predictions of how interfact roughness affects mirror performance. We are especially interested in studying how roughness evolves with polishing (in the case of mirror substrates) and with deposition (in the case of single or multilayer film deposition), and how thin film microstructure affects roughness.

One study, illustrated in Figure 11, investigates how substrate roughness evolves with polishing, and how the different levels of polishing affect the performance of multilayer mirrors deposited on those substrates. The substrates were fused-silica optical flats polished to four different levels of microroughness ranging from 0.2 to $11 \AA$ as determined by the polisher using visible interferometry. These roughness values span the range typical of most well polished $x$-ray mirrors, with the smoothest samples comparable to the best superpolished mirrors available. The $x$-ray reflectance and scattering measurements were made at the Stanford Synchrotron Radiation Laboratory using 1.38- $\AA$ radiation. Figures 11 (a) and (b) show the specular reflectance from the substrates alone and from an identical $W / C$ multilayer with $d=30.6 \AA$ deposited onto those substrates. Figure 11(c) shows diffuse scattering from the bare substrates 

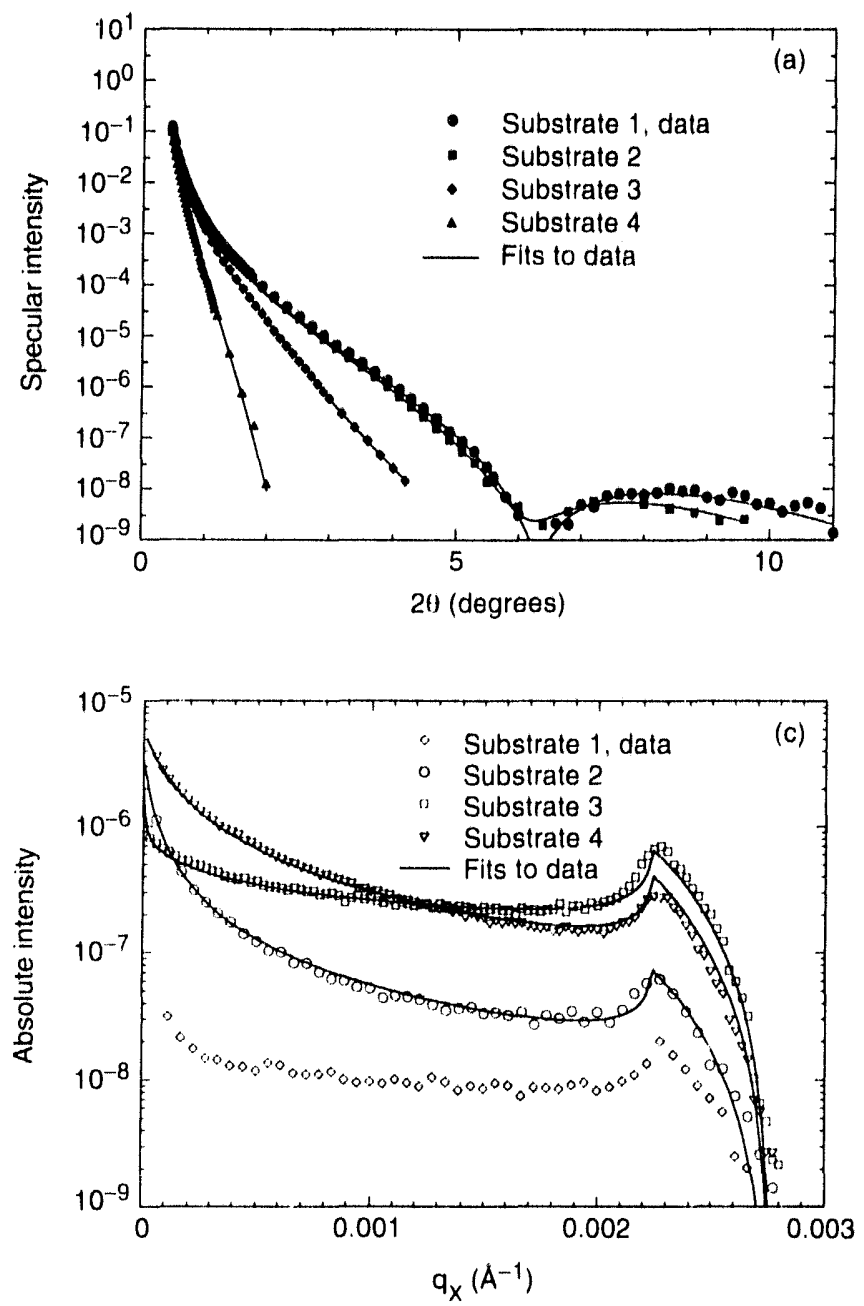

XBL. 937.4827

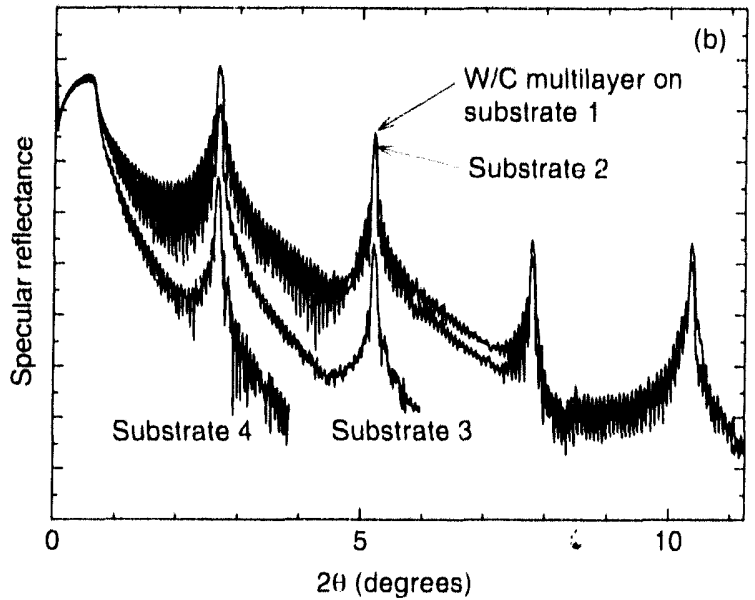

Figure 11. The specular reflectance $(\lambda=1.38 \AA)$ vs. angle is shown in (a) for a series of four fused silica optical flats polished to different levels of microroughness determined by the polisher with visible interferometry to be $0.2,2,5$ and $11 \AA$. (b) shows the reflectance from a W/C multilayer with 40 periods each $30.6 \AA$ thick. These data have had the diffuse component at the specular position subtracted to give the true specular intensily. Fits to the substrate data in (a) using a simple Fresnel model including a single overlayer are shown as lines through the data points, and yield roughness values larger than those obtained from visible interferometry. The reflectance at the first order multilayer peak decreases from $70 \%$, to $63 \%$, to $13 \%$ to $0.5 \%$ with increasing roughness, confirming that substrate roughness is a significant limitation to multilayer reflectance for all but ideally perfect substrates (ideal reflectance is $78 \%$ ). (c) shows the diffuse scattering measured from the bare substrates along $q_{x}$ (in the surface plane), together with theoretical fits using a model to describe scattering from fractally rough surfaces. The specular beam exists at $q_{x}=0$ and is not displayed here. The peaks at $q_{x} \equiv$ $0.0023 \AA^{-1}$ occur when the incident or reflected angles equal the critical angle, and indicate that the measured scattering results from surface not bulk fluctuations. The fractal model can fit scattering from all but the smoothest surface, whose scattering looks like white noise over most of this frequency range. The entire data set is consistent with growth of smooth $W$ and $C$ layers which conform successively to the roughness of the substrate over the range of frequencies measured.

measured with rocking scans, together with theoretical fits to these data using a model developed to describe scattering from fractally rough surfaces. Together the specular and diffuse scattering are extremely sensitive to the magnitude and nature of interface roughness.

This study demonstrates that, for high quality multilayers which form smooth and well-defined layers like the W/C multilayers studied here, multilayer reflectance is limited by substrate roughness. This limitation becomes increasingly severe as the multilayer period decreases, and can be the dominant factor limiting multilayers from achieving their theoretical ideal performance as wavelengths approach the $C$ edge and the water window. This study also emphasizes the reed for continued substrate development aimed at achieving ever smoother surfaces. 
Controlling Multilayer Period Variation for Imaging Applications

Figure 12. The need to control multilayer period $(d)$ variation on focusing optics arises because Bragg's law must be satisfied for each ray at each mirror at a fixed wavelength to have an achromatic imaging system. The primary and secondary mirrors of the 20x demagnifying Schwarzschild objective at the left present a range of angles that prescribe the ideal $/$ variations at the right. The colerance needed to match this prescribed variation of several percent is set by the multilayer bandpass, which decreases with wavelength to one percent or less at $68 \AA$ where $R u / B_{4} C$ multilayers have useful reflectance, We have developed techniques to specifically tailor the d variation on the curved surface of each mirror by introducing masks between the source and substrate to systematically reduce deposition much like dodging in the printing of a photograph.

Equally important is the ability to measure and verify these d variations using spatiallyresolved, near normal incidence reflectometry. The measured d variations are shown in the plots. The peak refleclance of the $R u /$ $B_{4} C$ multilayers across each mirror is roughly $15 \%$ at $68 \AA$.
Multilayer-coated focusing systems for XUV imaging, by their very nature, generally require laterally graded multilayer period or thickness across the clear aperture of mirrors to optimize performance. This requirement stems from the variation of incidence angles given by the optical design, and the inherent finite bandwidth of $x$-ray multilayers. The ideal period (d) variation at each mirror is prescribed for small-field imaging systems by the variation in incidence angle (from graving) across the clear aperture through the Bragg condition for constructive multilayer interference, $\lambda=2 d \sin \theta$. This is illustrated for a $20 \times$ demagnifying Schwarzschild objective in Figure 12, which shows the how the period should vary acress each mirror to avoid unwanted intensity and phase variations across the clear aperture. The actual d variation should conform to this prescribed ideal variation to a tolerance of a fraction of the multilayer band width.

The bandwidth of soft $x$-ray multilayers scales with wavelength, and hence so does the need to precisely control the multilayer period variation. $\mathrm{Mo} / \mathrm{Si}$ multilayers for use at $>125 \AA$ have bandwidths of $4-5$ percent or larger, leading to relatively generous tolerances for the control of $t$. Ru/ $B_{t} C$ multilayers have high reflectance at $>66 \AA$ and bandwidths of 1 percent or less, requiring the development of new techniques to control and verify the d variation on this and similar optics. These narrow bandwidths not only necessitate improved control of it variation across each individual curved surface, but also increase the demands on matching the coatings of different mirrors in a compound imaging system.

We have developed experimental technicues to control and verify the d variation of narrow band width multilayers on a $20 \times$ demagnifying Schwar/schild. Two issues are key in these developments, the ability to preciscly control d variation during deposition over steeply curved surfaces, and the ability to precisely measure the multilayer reflectance spectra with adequate spatial resolution on these curved surfices. We obtain control over depesition profile by introducing masks between the source and substrate to alter the depesition profile in a specifically desired manner. Spatially resolved measurement of id variation across these

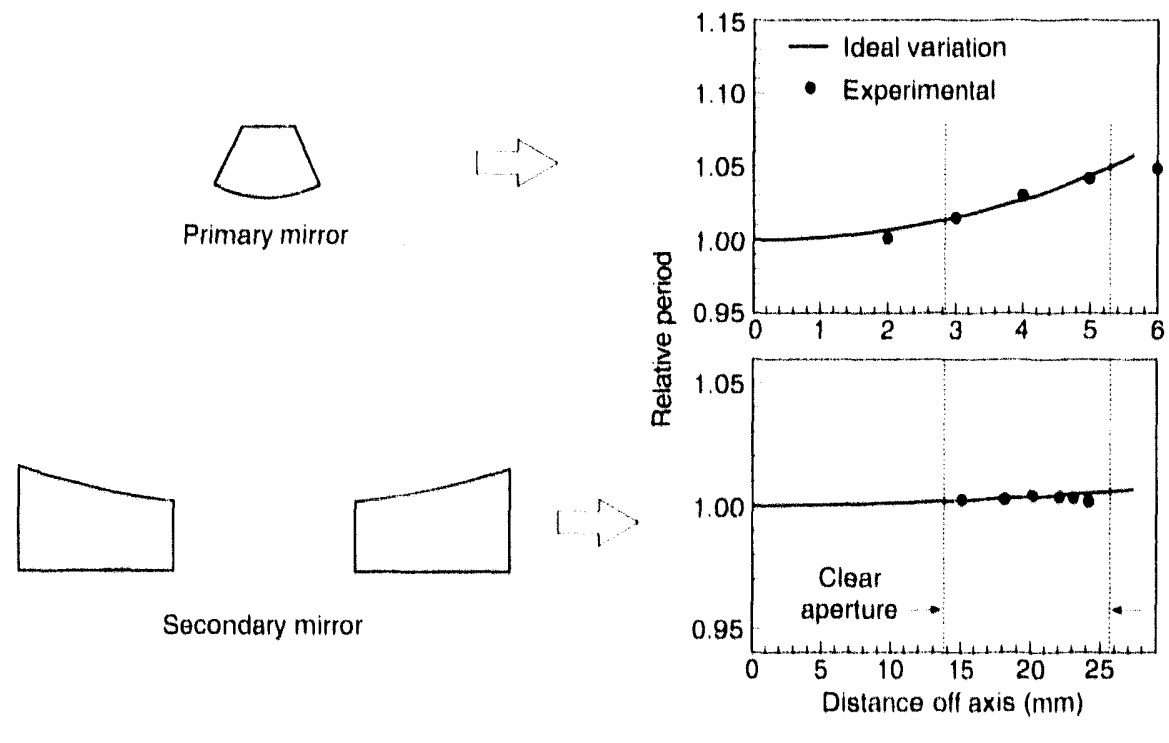

X日L 927.6755 
curved surfaces is accomplished with at-wavelength, near normal-incidence reflectance measurements using our newly developed laser-plasma based reflectometer. An iterative approach of deposition profile modification and spatially resolved reflectometry converges on an acceptable solution to the coating problem, and demonstrates unprecedented control of multilayer coatings on curved surfaces. These techniques are generally applicable to condenser and imaging optics of interest for soft $x$-ray projection lithography, as well as other multilayer-coated focusing systems.

Multilayer x-ray mirrors of molybdenum and silicon operating at normal incidence at wavelengths just longward of the $S i L_{11,113}$ absorption edges are a key component in the development of extreme ultraviolet projection lithography. Because the achievement and maintenance of high reflectivity surfaces are vital, it is important to know if Mo/Si multilayers change reflectivity with time, and a series of experiments was carried out to investigate this possibility. Mo/Si multilayer mirrors were prepared to reflect $148 \AA$ radiation at normal incidence with 50 layer pairs, and $d=76.2$ $\AA$. The reflectors were prepared both with molybdenum as the terminating layer (Mo on top) and with Si as the terminating layer (Si on top). The reflectivity of these multilayers on silicon wafers was measured immediately after deposition using the laser plasma reflectometer and then at intervals of a few days. Between measurements the samples were stored in laboratory air. The results are shown in figure 13. immediately after deposition both sets of mirror samples had a reflectivity of between 61 and $63 \%$, but over a period of months the reflectivity of the Mo-on-top samples decayed exponentially to an asymptotic value of about $48 \%$, while the Sion-top mirrors maintained their original reflectivity. Chemical analysis of the surface of the aged Mo-on-top mirrors showed that the topmost molybdenum layer had become oxidized to $\mathrm{MoO}_{3}$ and $\mathrm{MoO}_{2}$, and computational modeling showed that such oxidation could explain the drop in reflectivity. Since the observed decrease in reflectivity for the Mo-on-top mirror would lead to a decrease in throughput by a factor of 4 for a sevenelement projection lithography system, it is clearly preferable to fabricate the mirrors with silicon as the topmost surface.
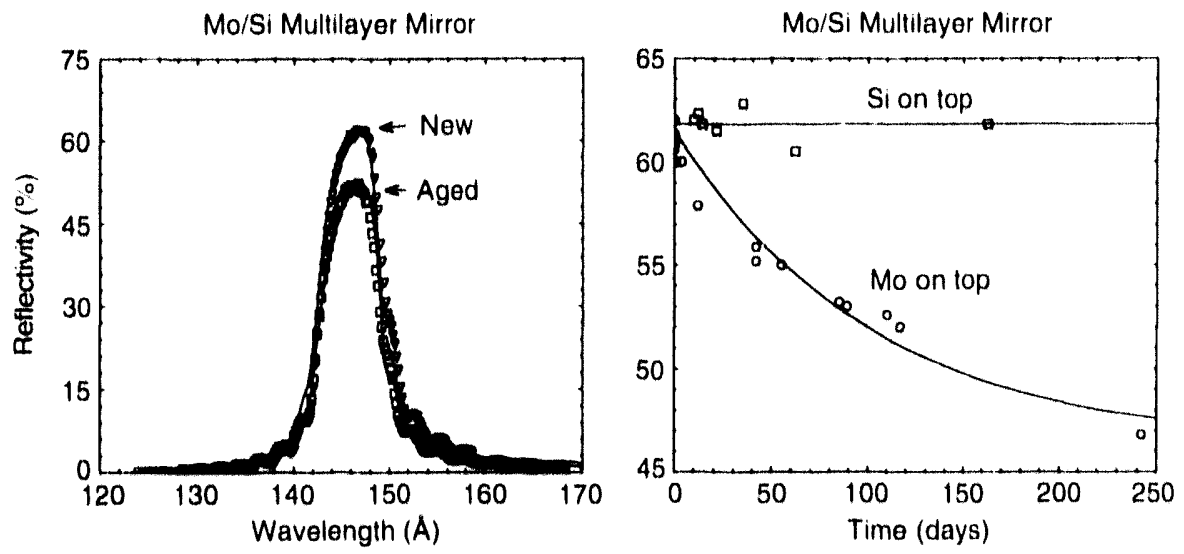

XBL. 835.4790
Tarnishing and

Restoration of $\mathrm{Mo} / \mathrm{Si}$ Multilayer X-Ray Mirrors
Figure 13. Lift: Reflectivity as a function of wavelength for a $\mathrm{Mo}$ / Si multilayer mirror designed to reflect $148 \AA$ at normal incidence. This mirror had Mo as the top layer; the two curves show the reflectivity just after deposition (triangles) and after storage in air for 244 days (squares). Right: peak reflectivity vs. time for the same multilayer compared with the peak reflectivity $\mathrm{v}$ s, time for an identical multilayer having silicon as the top layer. The silicon protects the molybdenum against oxidation, which is the cause of its degraded reflectivity. 


\section{Electron Beam Microanalysis of Light Elements}

Figure 14. The Ko line of nitrogen (a) with a commercially avallable layered dispersion elements (L.1)Es) with layer pairs of tungsten and silicon, and (b) with a new high reflectivity L.DE: developed at CXIRO. The peak signal is approximately 11 times greater for the CXR()-developed device.
(XRO) is developing multilayers that can be used in electron probe microanalysers to do cuantitative anolysis of light colements such as fluorine, oxygen, nitrogen, carbon, and lithium. In the past this has been difficult due to a lack of crystals with a lattice period d large enough to diffract the long wavelength characteristic $x$-rays from these elements. Mica, for cexample, with a period (d) of $19.8 X$, comnot be used for allalysis of oxygen, whose $K$ ex emission is at $23.4 \lambda$, or nitrogen $(33.2 \lambda)$, or carbon $(43.8 \AA)$. (On the other hand, multilayer reflectors con be mode with the repuired i-spacing and have been used as artificial crystals for microprobe analysits. Most have very low efficiency (1"in or less). Wo have developed improved layered dispersion olements (1.1) fis) with officiencies of $5 \%$ or more for these wavelengths. They allow the detection of the light alements with better signal to noise ratios and hence improved sensitivity. ligure it shows detection of the $K$ ox line of nitrogen (a) with a commercially av ailable l.1) E with layer pairs of tungsten and silicon, and (b) with a new high reflectivity L.DE developed at (XXRO). The prosk signol approximately 10 times greater for the (XRO) device. Similar results have been obtained in the analysis of oxygen. This improvement in light olement analysis capability has important applications in, for example, moterials science and geochemistry, and may provide particularly important opportunities for industrial development.
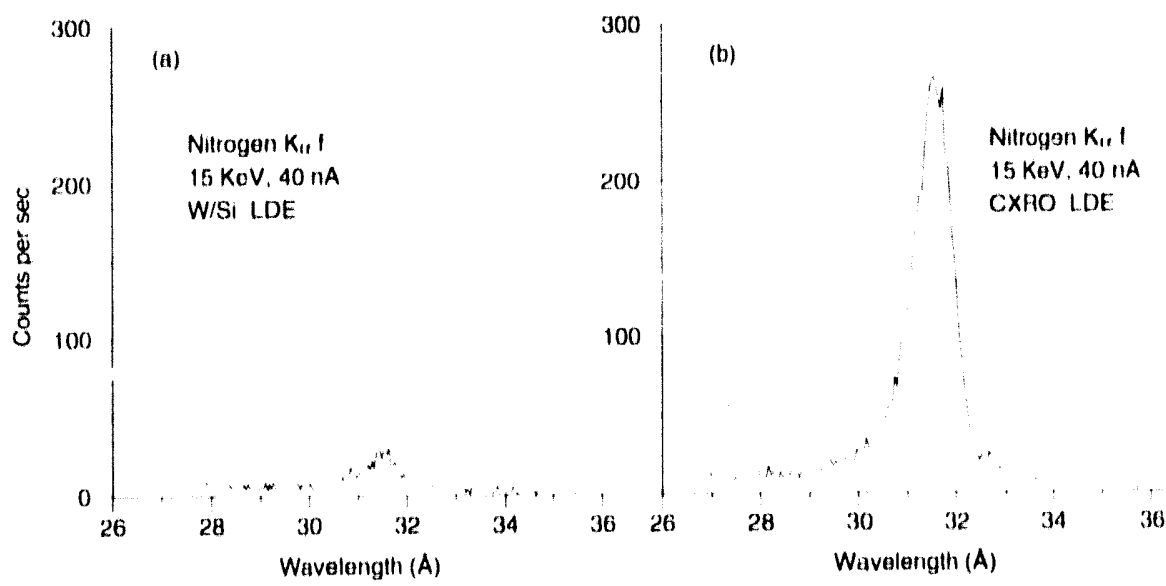

XIII. 9217.4628

\section{EUV / Soft X-ray Reflectometer}

The ability to make accurate and reliable calibration medsurements in the extreme ultravidelet/soft $x$-ray region is repuired lo enable scientists and engineers to measure the performance of optical elements and predice the performance of a system. This need is driven by the corrent activity in projection lithography as well as a variety of studies using synchrotron radiation. (Our present copability for making such moisurements centers around our reflectometer using a lasere-produced plasma EUV source, and shown in liggure 15. It usess a unigue high throughput monochromater designed and built at (XRO) that varies the wavelength to the optical alements being tested. Some recent studies, which include investigations of the stability of $\mathrm{Mo/Si}$ multilayer mirrors, and the development of multilayer contings for imaging optics at short wavelength are described in the multilayer section of this antabal report. 


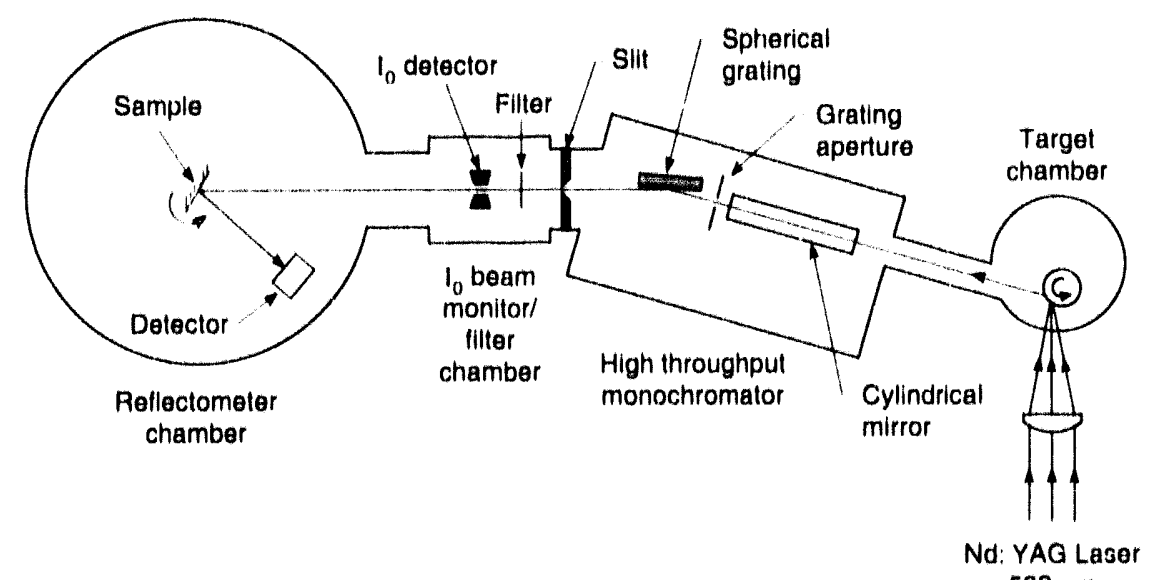

$532 \mathrm{~nm}$

XBL. $916.6759 \mathrm{~A}$

There is also a continuing need for improved measurements of the optical constants of materials in order to be able to predict and understand the performance of optics. Particularly, in the soft x-ray/extreme ultraviolet spectral range the optical constants are poorly known because of the difficulties involved in their measurement. We have obtained the optical constants for a variety of materials using both transmission and reflection measurement. (Our recent measurements of both the imagitiary (absorptive) and real (dispersive) parts of the index of refraction of platinum are shown in Figure 16, in which the data shown were obtained from the angle dependence of the reflectivity of a smooth platinum mirror. The measurements are compared to the indices of refraction as calculated from our compilation of atomic scattering factors described in a later section of this annual report.
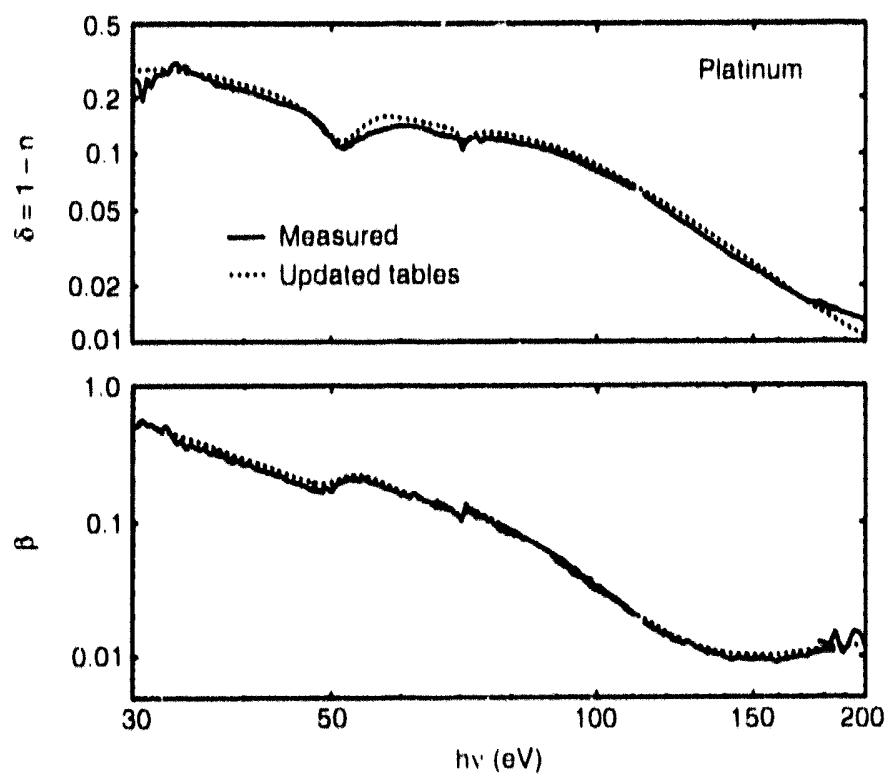

XBL. 937.4830
Figure 15. The CXRO extreme ultraviolet/soft $x$-ray reflectometer uses a laser-plasma source; the desired wavelength is selected from its broadband output with a unlque high-throughput monochromator designed and bullt at L.BL. (Conventional reflectometers use an $x$-ray lube of fixed wavelength.) The wavelength extends from about 30 (1, $1,1, \mathrm{~A}$.
Figure 16. The measured indices of refraction of platinum (solid lines) and the indices of refraction as calculated from our recent tabulation of atomic scattering factors (dashed lines). The measurement were oblained by angular dependent reflectivity using the reflectometer described above. 
Characterizations such as these will be performed with improved spectral and spatial resolutions and over a bronder photon energy range at a bending magnet beamline under construction at the AIS.S. The emphasis of these beamlines will be absolute measurements with moderate spectral $(\lambda \Delta \lambda \times 2(0) 0)$ and spatial resolution $(\Delta x=100) \mu \mathrm{m})$.

\section{Photoemission Microscopy with Reflective Optics}

Higure 17. MAXIMUM Photoemission Microscope as Installed at the University of Wisconsin's Synchrotron Radiation Center (SRC).
A collaboration between the Center for X-Ray Optics and the Synchrotron Radiation Center at the University of Wisconsin has produced the first scanning soft $x$-ray microscope using reflective optics. Working at a wavelength of $130 \AA$, the new microscope, shown in Figure 17, has demonstrated a spatial resolution of less than $0.1 \mu \mathrm{m}$ and simultaneously an clectron energy resolution of 350) millielectron volts (moV). This performance is significantly better than any achieved so far by alternative electron schemes. These developments are leading to a new generation of in understanding the heterengenenus nature of technologically and scientifically important surfaces on size scales ranging from several hundred angstroms upward. These features will become important as the Advanced light Source begins operation.

The Center for X-Ray Optics designed and fabricated the critical reflective multilayer interference conatings that allow normal incidence focusing at very soft x-ray wavelengths. Molybdenum/silicon multilayers having individual film thicknesse's of $3(1)-40) A$ were deposited onto the spherical surfaces of highly polished Schwarzschild objectives to provide reflectivities of o( 1 (1) per surface at $1.30 \AA$ wavelength. Special technicjues were developed to control the thickne'ss of the multilayer costings to ensure acceptable performance over the entire clear aperture of the optical system. The Center for X-Ray Optics also provided a custom designed monochromator and other beamline components to match the output of the undulater $x$-raly source to the micrescoupe objective.

ligure 18 shows an example of the manner in which the microseope may be used to study surfices with heterogene'ous chemical structure. The

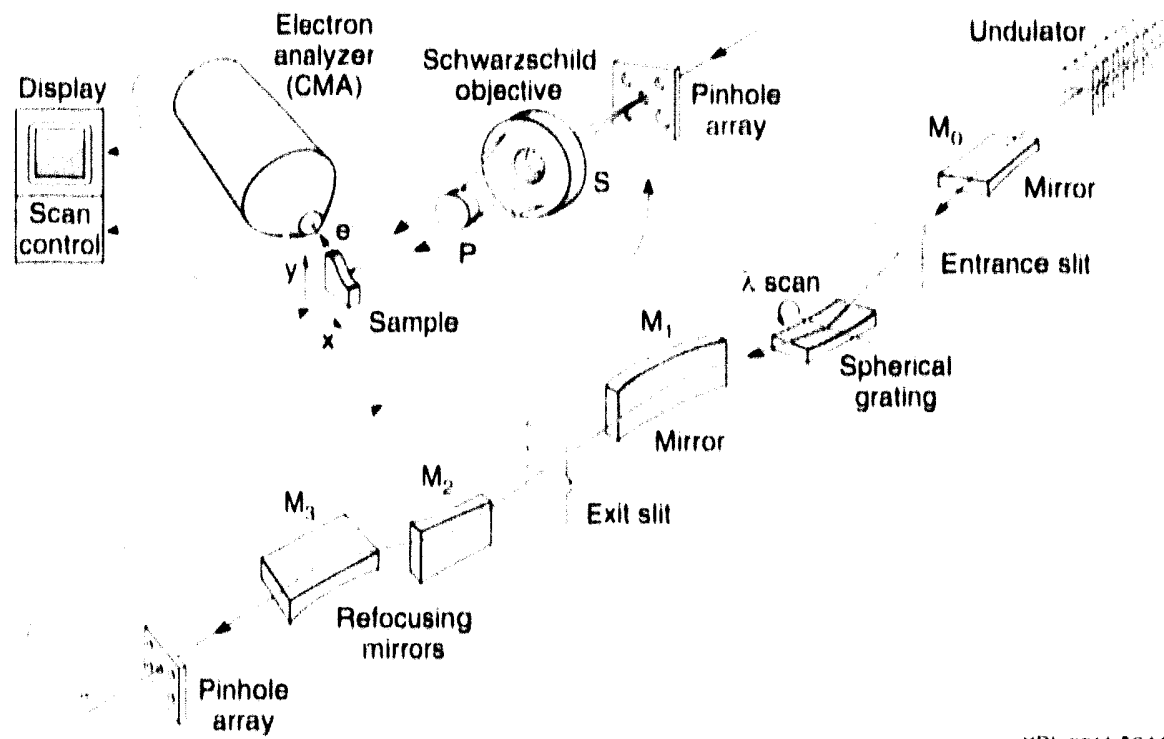
high spatial resolution surface analytical capabilities that will be important 


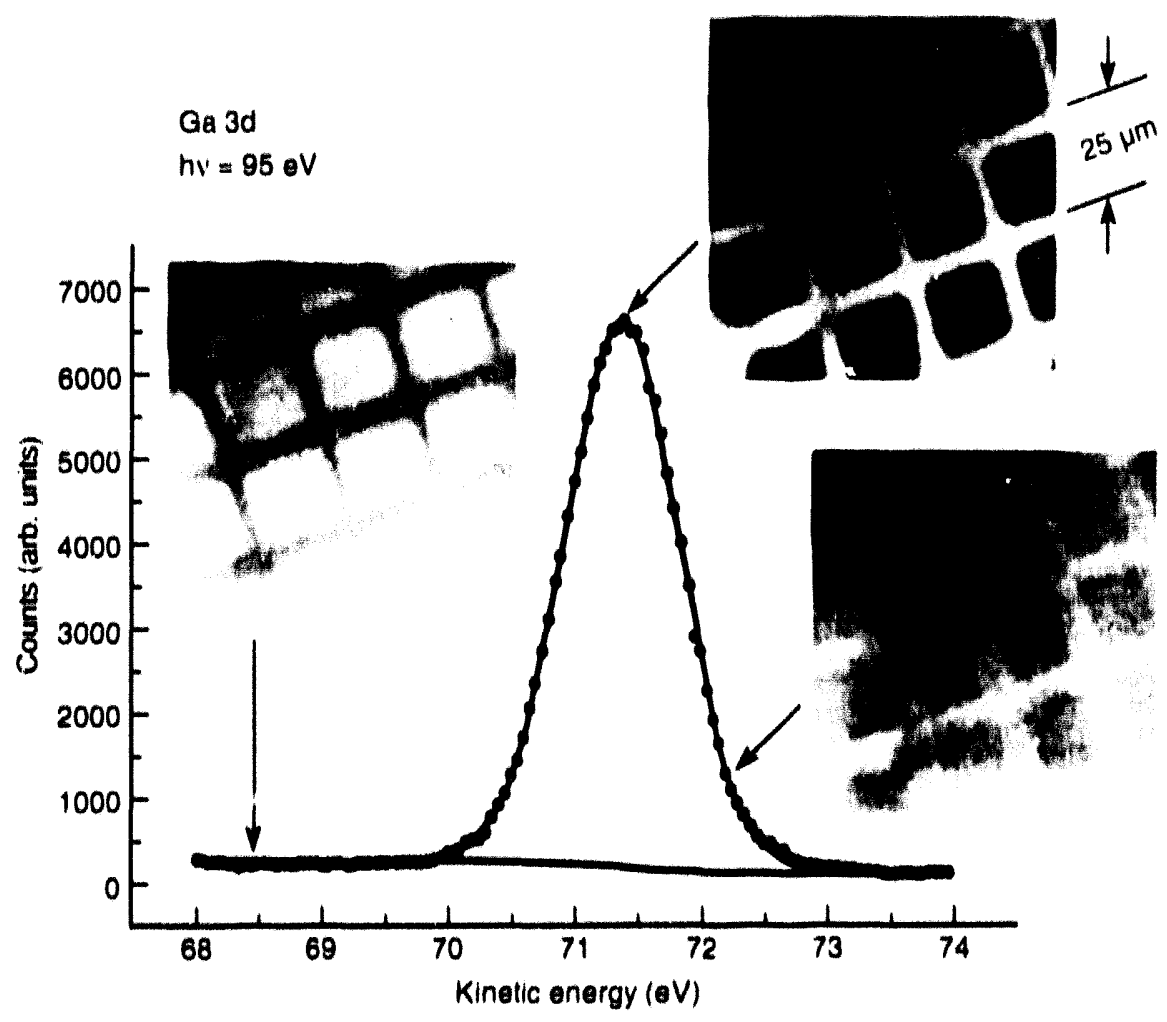

BaC 926.4890A

graph show's the energy distribution curve of the electrons emitted during a scan over the Ga 3 d core level taken on a cleaved gallium arsenide surface. The surface of the GaAs was masked with a $25-\mu \mathrm{m}$ mesh while 5() $A$ of gold was e'vaporated onto it, producing a pattern of gold squares. The inset images show spatial scans over $8(0) \mu \mathrm{m} \times 80) \mu \mathrm{m}$ areas of the specimen, using a $0.5 \mu \mathrm{m}$ spot, with the electron analyoer set at the three energies indicated by the arrows. The central image, set at the Gia $3 d$ peak, is essentially a map of the gallium distribution. The left-hand scan shows the map of the secondary electrons from the gold and is essentially a gold image, while the right-hand image is composed of a combination of the two.

The Center for X-ray Optics has always been involved in the design, construction and implementation of new types of $x$-ray and extreme ultraviolet spectroscopic instrumentation, both for synchrotron radiation research and for other applications. We have continued our efforts to develop new spectroscopic instrumentation with desirable properties such as high resolution, high throughput, simplicity and low cost.
Figure 18. The energy distribullon curve of the electrons emilted during a scan over the $\mathrm{Ga} 3 \mathrm{~d}$ core level taken on a cleaved gallium arsenide surface. Left insert: secondary electrons from gold squares. Center insert: $3 d$ electrons from gallium wire grid. Rlght insert: photoelectrons from both Ga and gold. MAXIMUM photo. emission microscope, Universily of Wisconsin, Madison.

\section{Spectroscopy with Soft X-Rays}


Monochromators and Spectrometers

Spatially and

Temporally Coherent Beamlines
To achieve high spectral resolution without compromising throughput, we have continued our investigations into monochromators, spectrometers, and spectrographs cusing plane gratlings with varied line spaceing. Such gratings allow a flat (or erect) focal plane and can thus be scanned in wavelength willout the complex scanning mechanisms required by moreaconventiomal designs, stich as sphorical grating monochromators. (One product of this work has been the High Resolution Streaked Spectrograph in use an the Nova laser at Lawrence L.ivermore National Laboratory. In addition, wo have continued our studies of atvanced monochromator and spectrometer designs for synchrotron radiation applications.

Recently we have developed computer codes that use ray-tracing fechnicyues fo simulate synchrofron radlation and estimate grating effi.

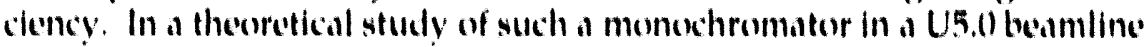
at the ALS, we confirmed that if would provide high spectral rewolution, high throughput, and small spols slas. To put these results fo the fest af experiment, we are planning to bulld a new varied-line-spacing planegratting monochromator for tose at BLSSY.

In another application-oriented program, we are investigatting design options for dividing a soft x-roy beom among sovernl users. This is chol. lenging beciluse an indulater senterce in a modern, low-emithance storage ring puts ont a very thin, yuastamonoshromatic beam that does not lond Itsolf readily to oither spatiol or spectral splitting. The possibilities that we are examining include. wavefront splitting, high-frepuency timesharing, and splitting according to diffractionsegratting order. The fladings will be

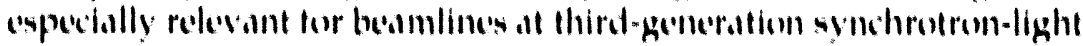

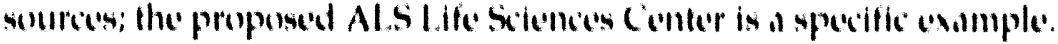

An unduhator having a long poriodic mognetic structure installed at a

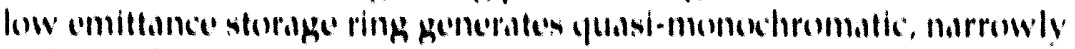
collimated, and partially coherent radiation. Howevere, unduhators car-

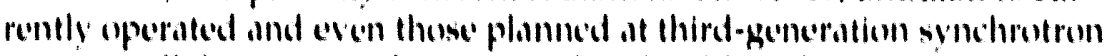

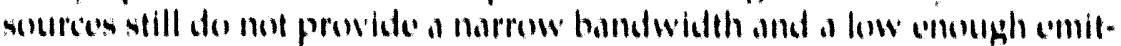

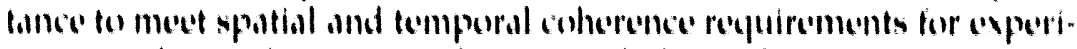
ments such as sofl saray inferferometry, hologrophy, and scaming microse

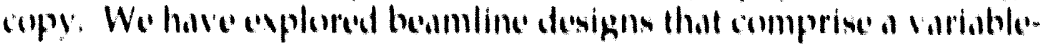

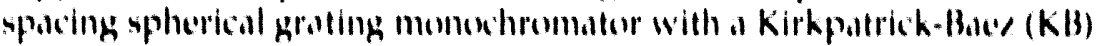
prefocusing system to dediver a spottally and temponally cohereme flus info

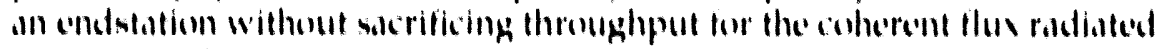
from the undulater. The designed system is schemotically shown in figure 19. The first mirror is a pepe-14p plane mirror for swite hing the ineoming

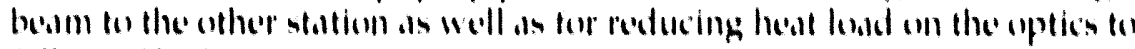

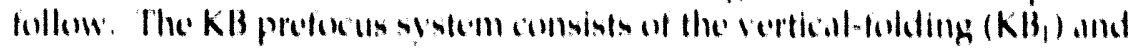

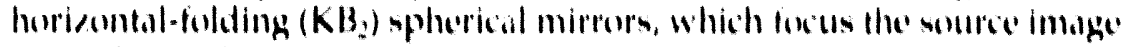

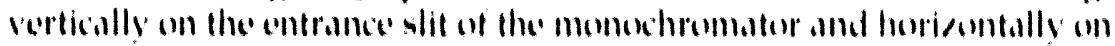
the exit slit (pinhole) respectively. The pinhole situited at the plame of the exit slit and the apertare in the endstalten define the through-roses in the

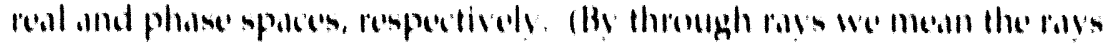
Hot poss through both the pinhole and the aperture.) The prodect al

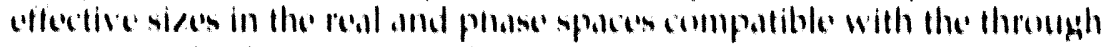

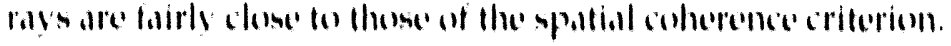




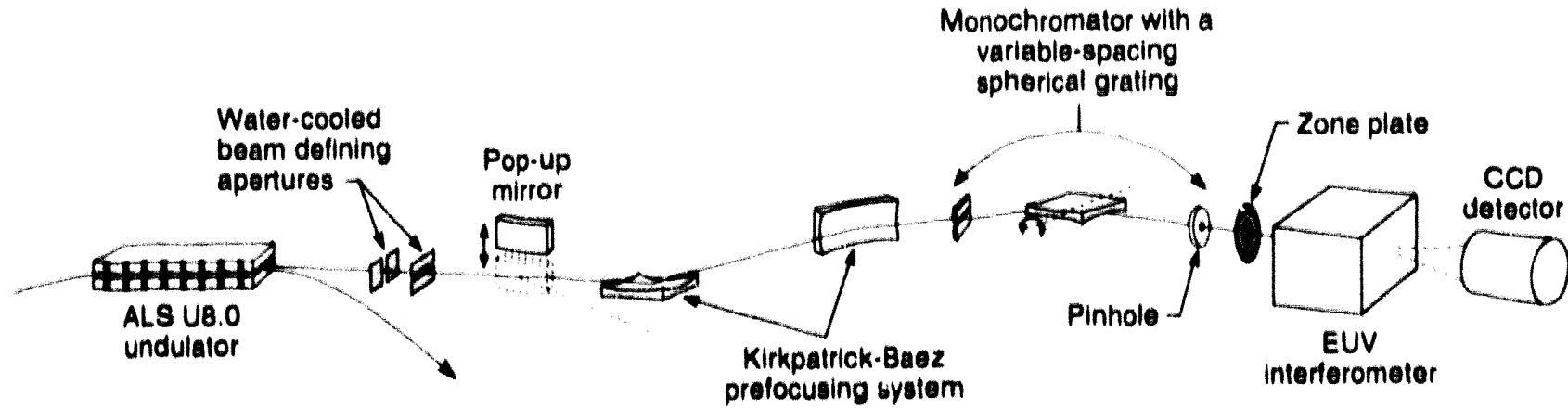

Xth 937.462

Figure 19. Coherent radiation beamline consioting of a pop-up mirror, Kirkpatrick-Bace

(KB) prefocusing mirrors, a varlable spacing spherical grallng monochromalor, a pinhole. lens spatial fllier, and an endslation. Beamlines such an this will be used for scanning v-ray microscopy, "al-wavelength" Interlerometry, and other applications requiring coherent BUV or solf-x-ray radiation.

Our design offorts have been extended to a high resolution VUV monechromator and its beamline to be deverted to chemical dynomic studies. The exporimental syatem consists of a VUV high resolution monochromator beamline, infrared lasers, and molecular beam chambers (sec Higure 20). The scientific research program will focus on gaining a rigorous molecular-level understanding of combustion and other energetic processes. An intense undulater rodlation focused on a denuous molecular beam enables one to observe transient molecules having very short life-

\author{
High Resolution VUV \\ Menochromator for the \\ Chemical Dynamics \\ Heamline
}

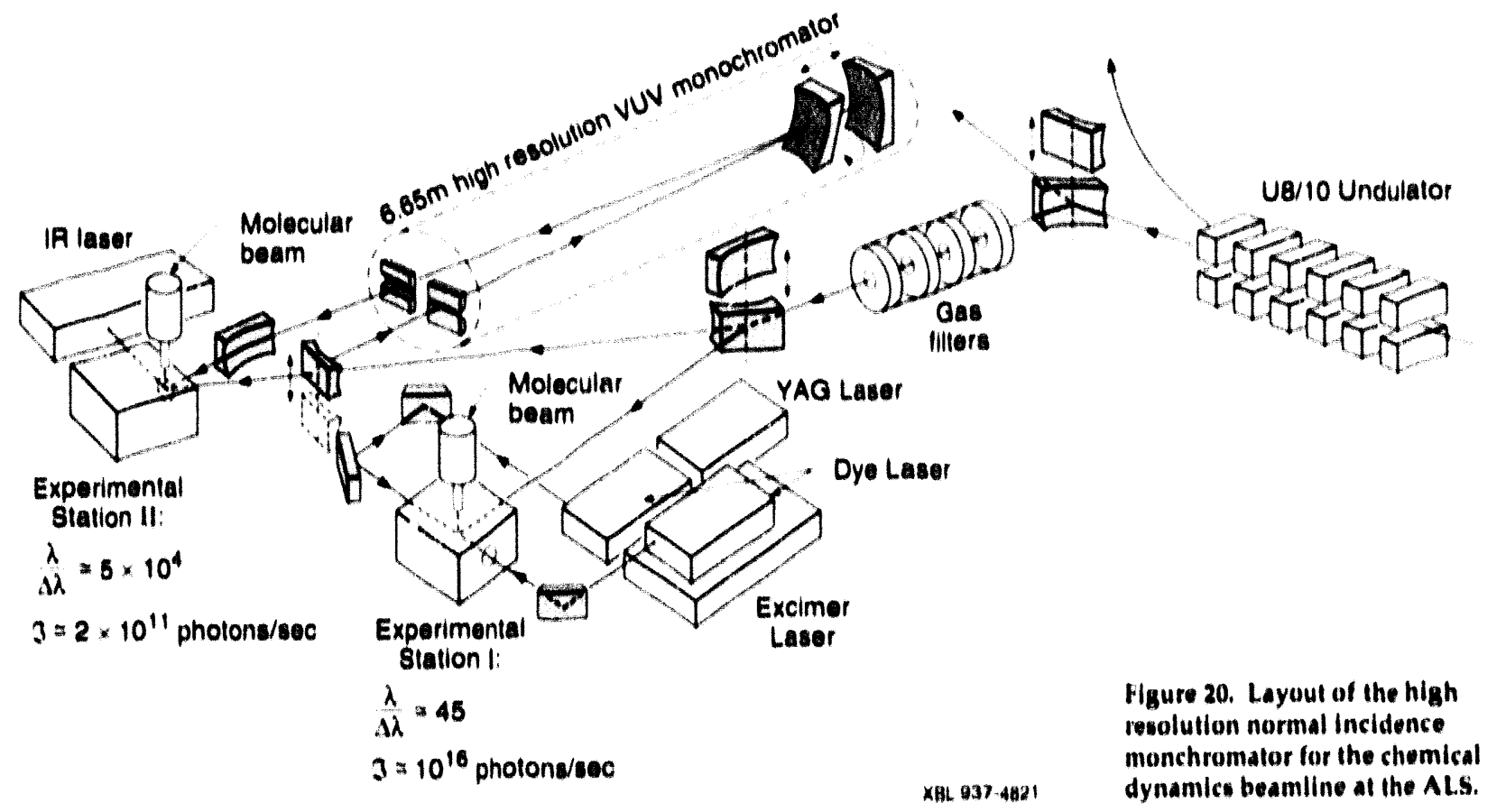




\section{High Reisolutions \\ Monochromator for Cirbon-Roluted Studien}

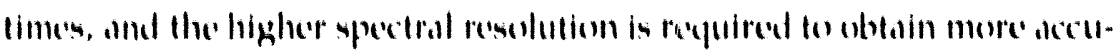

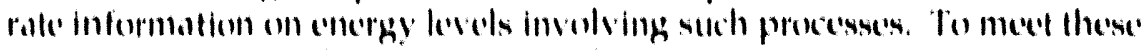

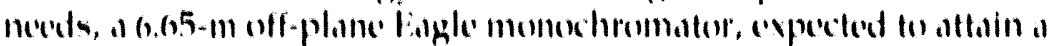

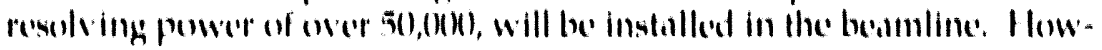

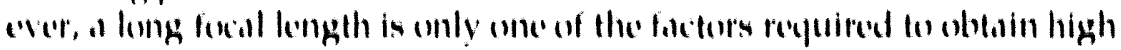
resulution with high throughiput. (Mhere fictors include use of a gratting

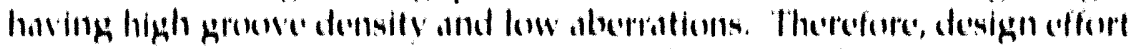
has been placed an compatibility between lhe various kinds of gratings

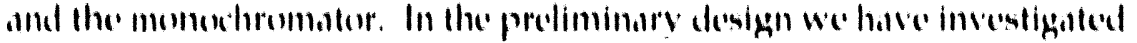

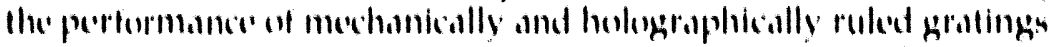

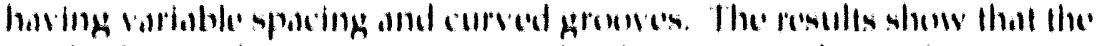

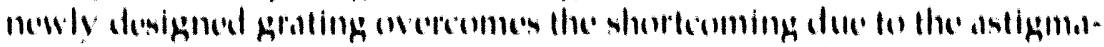

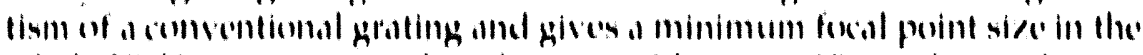

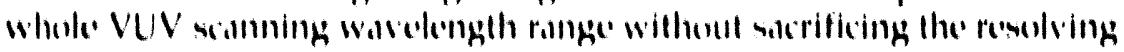
puintr.

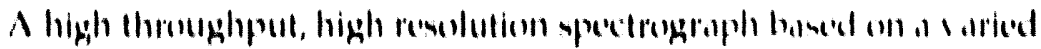

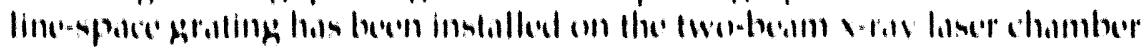

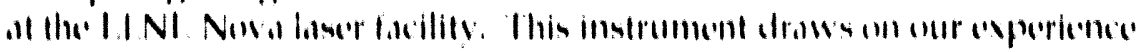

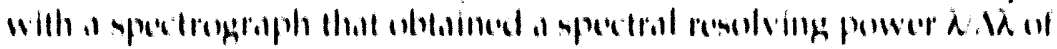

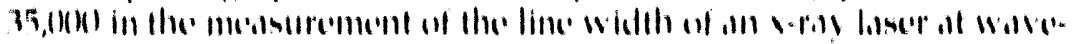

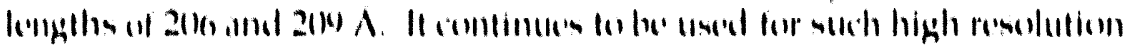

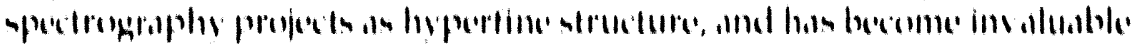

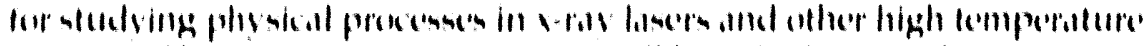

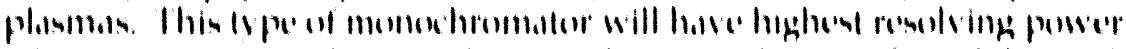

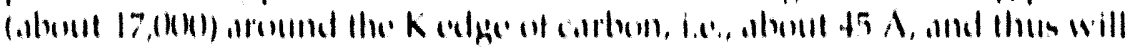

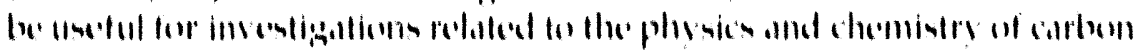

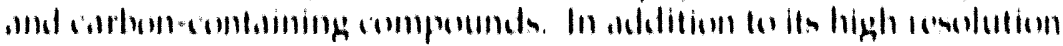

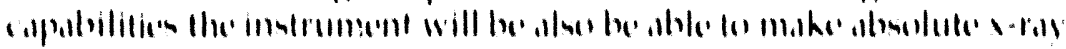

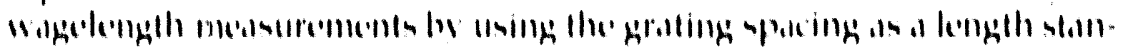

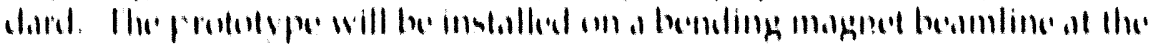

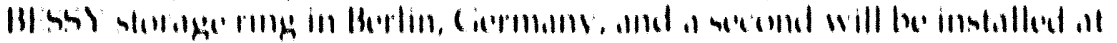

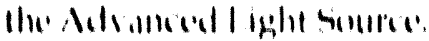

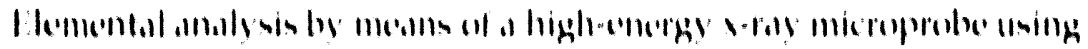

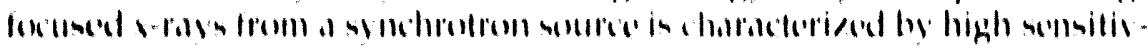

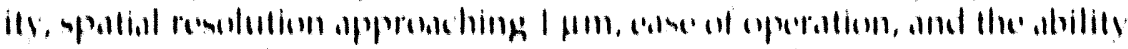

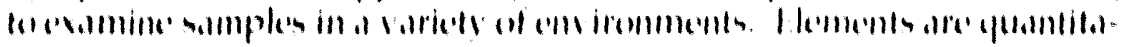

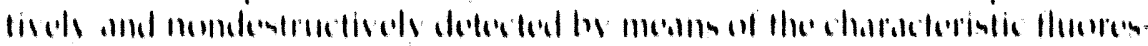

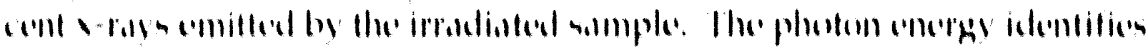

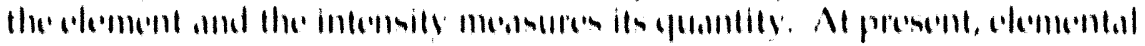

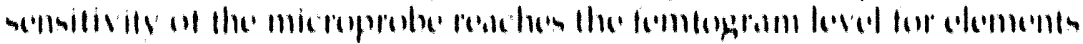

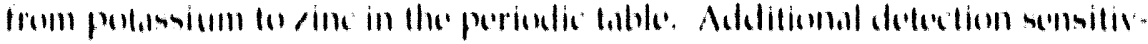

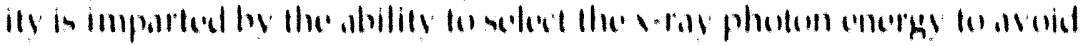

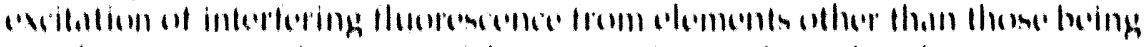

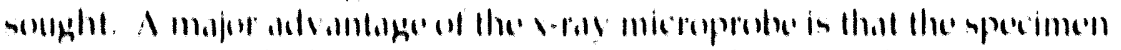

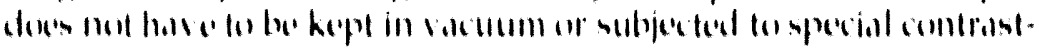

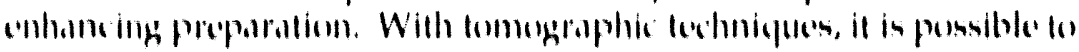

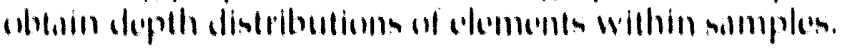




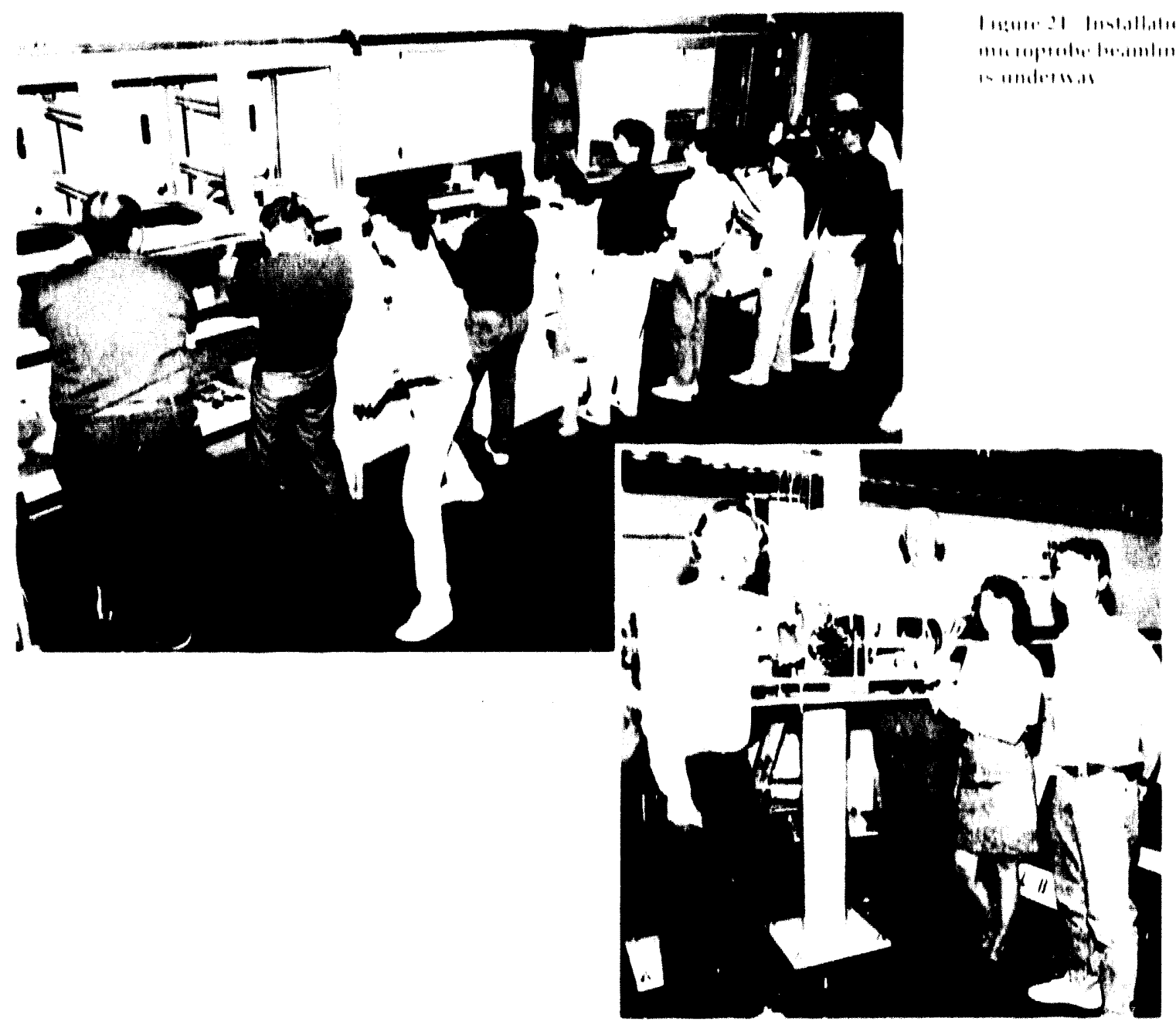

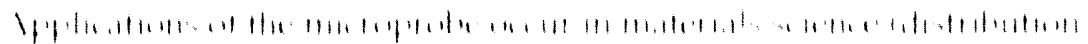

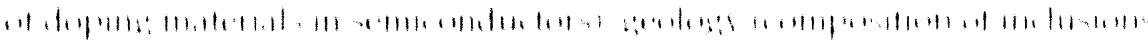

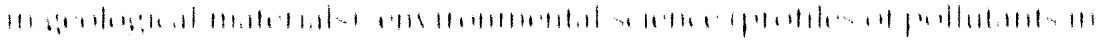

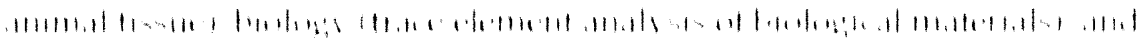

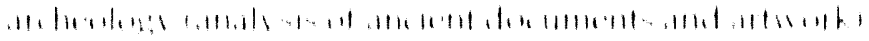

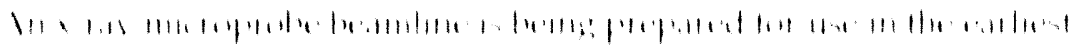

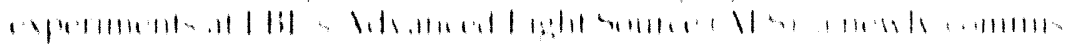

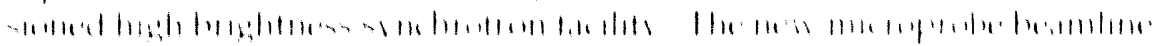

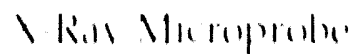

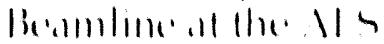

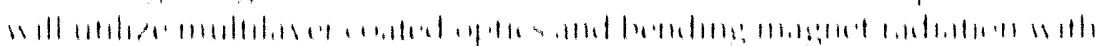

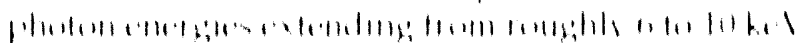

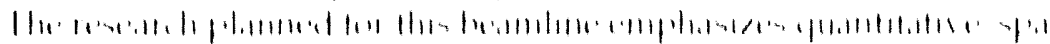

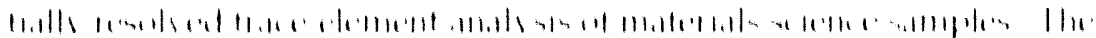

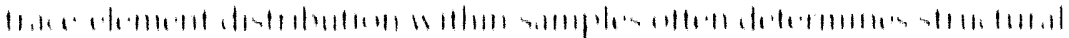

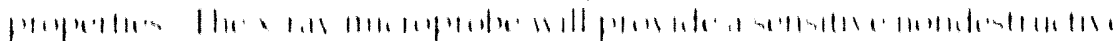


fool for two-dimensional distribution of trace elements with a spatial resolution of $1 \mu \mathrm{m}$. The microprobe will be particularly useful for the characterization of defects and cracks in ceramic materials. Of particular interest are (1) cleavage or ductile fracture in bulk ceramic, such as partially stabilized zirconia, alumina, silicon carbide, and silicon nitride, or in ceramic composites, such as silicon carbide in alumina, (2) subcritical crack growth by stress corrosion or fatigue, and (3) fracture at the ceramic metal interface in metal-matrix composites, such as $\mathrm{SiC}-\mathrm{Al}_{2} \mathrm{O}_{3}$. It is particularly important to determine whether crack processe's occur in the bulk or only at the surface. The movement of different elements during annealing, mechanical stress, and chemical processing will also be studied.

The Advanced l.ight Source (ALS) is a national facility for scientific research and development, where work may be conducted as a member of a participating research team ('R'T). The microprobe l'RT consists of members of the Materials Sciences, Applied Sciences, Barth Sciences, and Engineering Sciences divisions of I. BI. as well as members from the Oak Ridge National Laboratory and the Lawrence Livermore National Laboratory. Beamline 10.3 will have two white-radiation branches. One experimental station will be dedicated to materials science experiments; the other will be used to develep advanced $x$-ray optical componerits and instrumentation.

\section{Coronary Angiography}

X-ray beams from a synchrotron source-monochromatic, wellcollimated, and intense-provide uniçue opportunities for medical imaging. (ne of them is a new method of coronary angiography that use's venous injection of contrast agent, as opposed to arterial injection. For several years we have participated in a collaboration that is working on this new, safer method. A new step toward large-scale tests of clinical applicability was taken in 1990 with the completion of a dedicated medical-imaging facility on a special wiggler beamline at the NSISS. V:leven patients' hearts have been imaged there; the results are not yet equivalent to conventional angiograms, but they are approaching clinical usefulness, and the technique is dramatically casier and safer for the patients.

This year the imaging system at the NSI.S was upgraded in two ways: A new latue monochromater was installed, which increased the available $x$-ray flux by a factor of 8 over the previous monochromator. A new I.BI.built detecter with 1200 elements was also installed that replaced the previous 60)(-edement detector. The computer system and data-acyuision software were also upgraded. The new system was tested in September 1993, and will be used with patients in early 1994.

Images obtained with the old system thus far clearly indicate that large portions of both the left anterior and the right corenary arteries can be examined with this method. Various changes are now underway to further improve the image yuality. Then a medical research team will begin to use this technigue on a large group of patients, comparing the new methed to standard coronary angiography and also studying the effect of various medical treatments on the progression of coronary artery disease. An example of the image thus far acheived is shown by figure 22. 

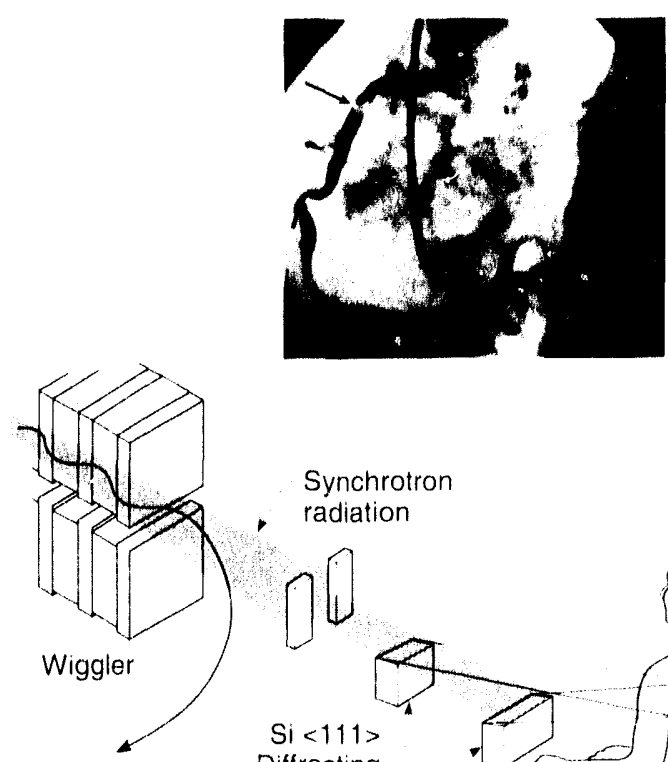

Electron Beam

Synchrotron

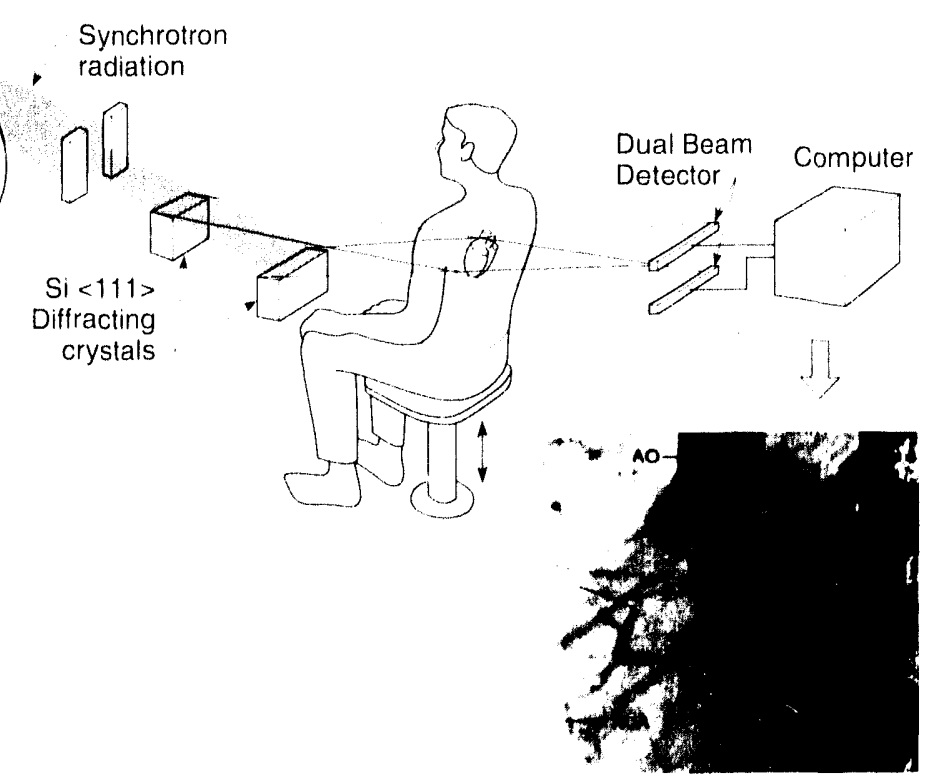

XBB 937-5003A

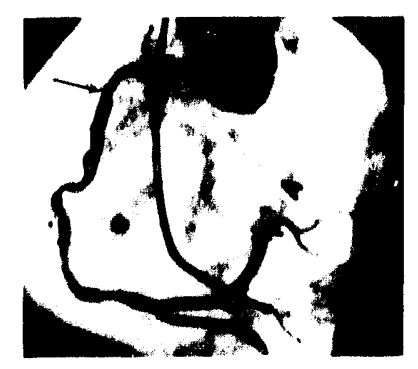

An extended tabulation of the atomic scattering factors, which describe the basic interaction of $x$-rays with matter, has been completed this past year, and is scheduled to be published in a special issule of the Alomic Data and Nuclear Data Tables in July 1993. These tables represent a revision of the 1982 compilation by Henke et al., which are widely used throughout the $x$-ray community. The revision is based on experimental measurements that have been published during the past 10 years as well as improved theoretical calculations. The range is extended to cover photon energies from 50 to $30,000 \mathrm{eV}$ for the elements $Z=1$ to 92 . The are also being freely distributed on computer disk.

In order to improve our knowledge of the atomic scattering factors, better measurements will be required. Such data are currently obtained from measurements of the transmission through a thin film or the reflection from a smooth surface, or by $x$-ray interforometry. Measurements have been periormed using our present sources (see the XUV Reflectometer section) and will be extended with the use of beamlines now under construction at the ALS.

Figure 22. Angiograms made with a standard arterial injection of contrast agent, an invasive and relatively dangerous procedure, are shown at top left and right. At the lower right is an angiogram of the same patient taken with the synchrotron-radiation method, which uses a much less hazardous venous injection. The left image was taken just before a balloon angioplasty uperation to clear a blockage (see arrow in image) in the right coronary artery (RCA). The top right image was taken just after the operation and the lower right image was taken 6 months later. The image quality of the lower right image, while not yet as good as the upper two images, shows clearly that coronary arteries can be clearly seen with the less invasive venous injection method. The significantly reduced hazard may permit studies of the progression of heart disease, and might some day be useful for clinical studies. 


\begin{tabular}{|c|c|c|c|c|c|}
\hline Line & $\mathrm{E}(\mathrm{eV})$ & $\mu\left(\mathrm{cm}^{2} / \mathrm{gm}\right)$ & $f_{1}$ & $f_{2}$ & $\lambda(A)$ \\
\hline $\mathrm{H}$ & 10.2 & $9.03 e+4$ & & 2.10 & 1215 \\
\hline He I & 21.2 & $1.50 \mathrm{e}+5$ & & 7.27 & 584.3 \\
\hline $\mathrm{Na} \quad \mathrm{L}_{2,3}$ & 30.5 & $5.08 e+4$ & & 3.53 & 407.2 \\
\hline $\mathrm{Mg} \mathrm{L} \mathrm{L}_{2,3}$ & 49.3 & $1.26 e+5$ & & 14.17 & 251.5 \\
\hline Al $\quad L_{2,3}$ & 72.4 & $2.47 e+4$ & 17.21 & 4.08 & 171.2 \\
\hline $\mathrm{L}_{2,3}$ & 91.5 & $7.03 e+3$ & 14.96 & 1.47 & 135.5 \\
\hline $\mathrm{Be} \mathrm{K}$ & 108.5 & $4.36 e+3$ & 13.32 & 1.08 & 114.3 \\
\hline $\mathrm{Sr} \quad \mathrm{M} \zeta$ & 114.0 & $4.37 e+3$ & 12.88 & 1.14 & 108.8 \\
\hline $\mathrm{M} \zeta$ & 132.8 & $4.94 e+3$ & 11.87 & 1.50 & 93.4 \\
\hline $\mathrm{Zr} \quad \mathrm{M} \zeta$ & 151.1 & $5.57 e+3$ & 11.12 & 1.92 & 82.1 \\
\hline $\mathrm{K} \alpha$ & 183.3 & $5.71 e+3$ & 9.94 & 2.39 & 67.6 \\
\hline Mo $\mathrm{M} \zeta$ & 192.6 & $5.60 e+3$ & 9.45 & 2.46 & 64.4 \\
\hline Ar L $\ell$ & 220.1 & $5.70 \mathrm{e}+3$ & 6.86 & 2.86 & 56.3 \\
\hline $\mathrm{K} \alpha$ & 277.0 & $2.14 e+4$ & 4.54 & 13.50 & 44.8 \\
\hline Ag $\mathrm{M} \zeta$ & 311.7 & $2.37 e+4$ & 7.56 & 16.85 & 39.8 \\
\hline $\mathrm{K} \alpha$ & 392.4 & $2.45 e+4$ & 14.36 & 21.89 & 31.6 \\
\hline $\mathrm{Ti} \quad \mathrm{L} \alpha$ & 452.2 & $2.19 e+4$ & 20.06 & 22.61 & 27.4 \\
\hline $\operatorname{L} \alpha$ & 511.3 & $1.91 e+4$ & 24.12 & 22.32 & 24.2 \\
\hline $\mathrm{K} \alpha$ & 524.9 & $1.85 e+4$ & 25.11 & 22.13 & 23.6 \\
\hline $\mathrm{Cr} \operatorname{L} \alpha$ & 572.8 & $1.59 \mathrm{e}+4$ & 28.23 & 20.70 & 21.6 \\
\hline$M n \operatorname{L} \alpha$ & 637.4 & $1.26 e+4$ & 30.36 & 18.34 & 19.5 \\
\hline $\mathrm{K} \alpha$ & 676.8 & $1.13 e+4$ & 31.17 & 17.41 & 18.3 \\
\hline $\mathrm{Fe} \quad \mathrm{L} \alpha$ & 705.0 & $1.05 e+4$ & 31.74 & 16.83 & 17.6 \\
\hline Co L $\alpha$ & 776.2 & $8.77 e+3$ & 33.11 & 15.53 & 16.0 \\
\hline $\mathrm{Ni} \quad \mathrm{L} \alpha$ & 851.5 & $7.12 \mathrm{e}+3$ & 34.34 & 13.82 & 14.6 \\
\hline $\mathrm{Cu} \quad \mathrm{L} \alpha$ & 929.7 & $5.80 e+3$ & 34.90 & 12.30 & 13.3 \\
\hline $\mathrm{Zn} \quad \mathrm{L} \alpha$ & 1011.7 & $4.84 e+3$ & 35.19 & 11.17 & 12.3 \\
\hline $\mathrm{Na} \quad \mathrm{K} \alpha$ & 1041.0 & $4.54 e+3$ & 35.34 & 10.78 & 11.9 \\
\hline $\mathrm{Ge} \operatorname{L} \alpha$ & 1188.0 & $3.35 \mathrm{e}+3$ & 35.68 & 9.07 & 10.4 \\
\hline $\mathrm{Mg} \mathrm{K} \alpha$ & 1253.6 & $2.96 \mathrm{e}+3$ & 35.73 & 8.46 & 9.9 \\
\hline $\mathrm{Al} \quad \mathrm{K} \alpha$ & 1486.7 & $1.98 \mathrm{e}+3$ & 35.61 & 6.71 & 8.3 \\
\hline $\mathrm{K} \alpha$ & 1740.0 & $1.35 \mathrm{e}+3$ & 35.07 & 5.36 & 7.1 \\
\hline $\mathrm{Zr} \quad \mathrm{L} \alpha$ & 2042.4 & $9.11 e+2$ & 33.83 & 4.24 & 6.1 \\
\hline Mo $\operatorname{L} \alpha$ & 2293.2 & $6.84 \mathrm{e}+2$ & 31.73 & 3.57 & 5.4 \\
\hline $\mathrm{Cl} \mathrm{K} \alpha$ & 2622.4 & $2.20 \mathrm{e}+3$ & 27.66 & 13.13 & 4.7 \\
\hline $\mathrm{Ag} \quad \mathrm{L} \alpha$ & 2984.3 & $2.02 e+3$ & 35.52 & 13.78 & 4.2 \\
\hline $\mathrm{Ca} \mathrm{K} \alpha$ & 3691.7 & $1.20 \mathrm{e}+3$ & 40.27 & 10.06 & 3.4 \\
\hline $\mathrm{Ti} \quad \mathrm{K} \alpha$ & 4510.8 & $7.12 \mathrm{e}+2$ & 41.71 & 7.32 & 2.7 \\
\hline $\mathrm{K} \alpha$ & 4952.2 & $5.57 e+2$ & 41.99 & 6.28 & 2.5 \\
\hline $\mathrm{Cr} \mathrm{K} \alpha$ & 5414.7 & $4.39 e+2$ & 42.14 & 5.42 & 2.3 \\
\hline $\mathrm{Mn} \mathrm{K \alpha}$ & 5898.8 & $3.49 \mathrm{e}+2$ & 42.19 & 4.69 & 2.1 \\
\hline Co $\mathrm{K} \alpha$ & 6930.3 & $2.25 \mathrm{e}+2$ & 42.13 & 3.56 & 1.8 \\
\hline $\mathrm{Ni} \quad \mathrm{K} \alpha$ & 7478.2 & $1.83 e+2$ & 42.07 & 3.12 & 1.7 \\
\hline $\mathrm{Cu} \mathrm{K} \alpha$ & 8047.8 & $1.50 \mathrm{e}+2$ & 41.99 & 2.75 & 1.5 \\
\hline $\mathrm{Ge} \mathrm{K} \alpha$ & 9886.4 & $8.50 \mathrm{e}+1$ & 41.72 & 1.92 & 1.3 \\
\hline $\mathrm{K} \alpha$ & 14988.0 & $2.67 e+1$ & 40.91 & 0.91 & 0.8 \\
\hline Mo $\mathrm{K} \alpha$ & 17479.0 & $1.74 \mathrm{e}+1$ & 40.36 & 0.70 & 0.7 \\
\hline$P d K \alpha$ & 21177.0 & $6.97 e+1$ & 39.98 & 3.37 & 0.6 \\
\hline $\mathrm{Sn} K \alpha$ & 25271.0 & $4.37 e+1$ & 41.59 & 2.52 & 0.5 \\
\hline $\mathrm{Xe} K \alpha$ & 29779.0 & $2.80 e+1$ & 42.02 & 1.90 & 0.4 \\
\hline
\end{tabular}
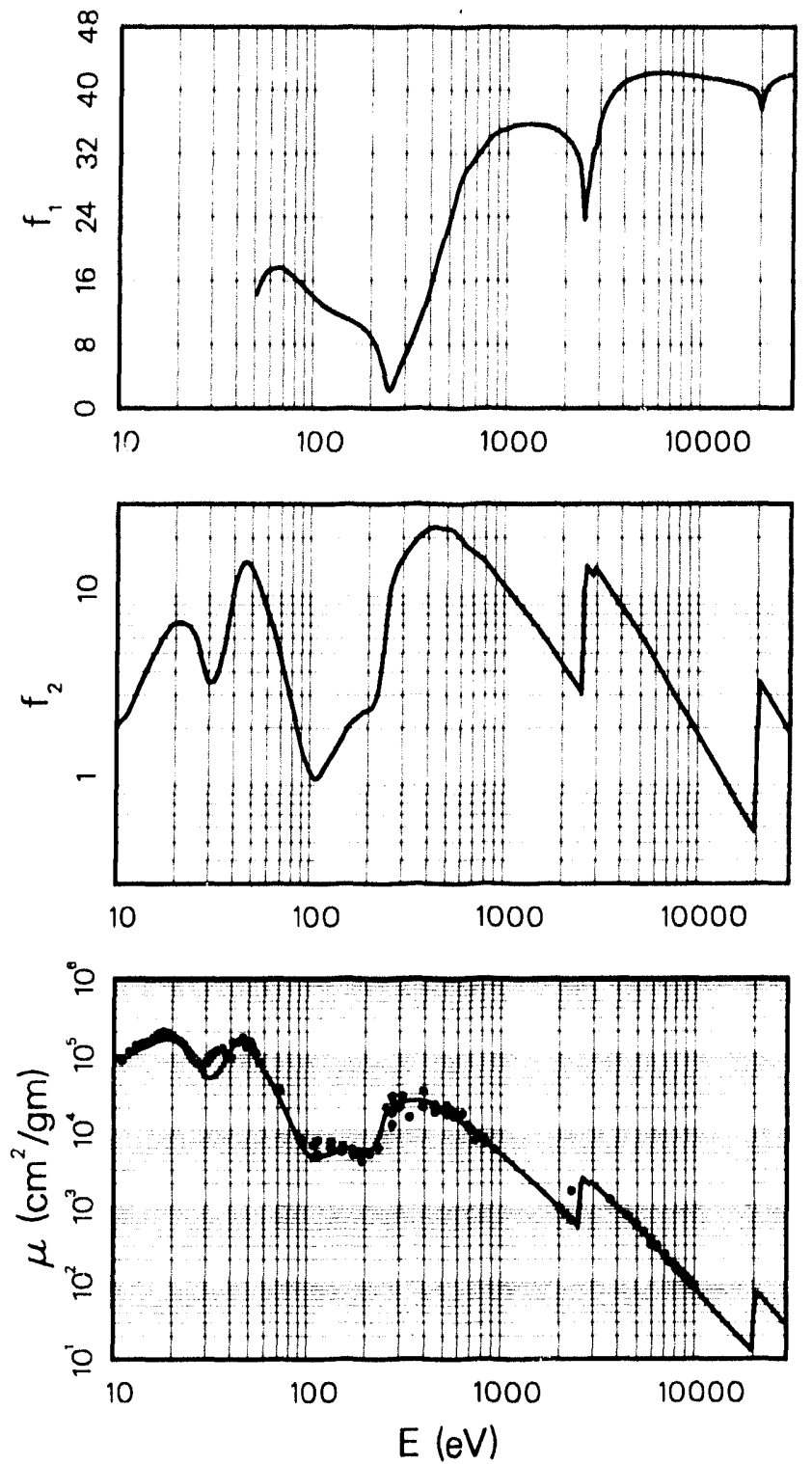

References: $33,48,52,59,76,115,123,127,131,177,200,205,223,233$.

Figure 23. The atomic scattering factors for molybdenum as will appear in the special issue of Atomic Data and Nuclear Data Tables in July 1993. This tabulation covers the extended range of 50 to $30,000 \mathrm{eV}$ for all elements from hydrogen $(Z=1)$ to uranium $(Z=92)$. 


\section{CENTER FOR X-RAY OPTICS}

Ade, $\mathrm{H}_{1}$, C.H. Ko and E. Anderson, "Astigmatism Correction in X-RayScanning Photoemission Microscope with Use of Elliptical Zone Plate," App. Phys. Lett., 60, 1040) (1992).

Ade, H., C.H. Ko, E.D. Johnson and E. Anderson, "Improved Images with the Scanning Photoelectron Microscope at the National Synchrotron Light Source," Surface and litterface Anolysis, 19, 17 (1992).

Anderson, E.H., V. Boegli, M.L. Schattenburg, D. Kern and H.I. Smith, "Metrology of Electron-Beam Lithography Systems Using Holographically Produced Reference Sample," I. of Vac. Sci. E Tech, B, 9, 3606 (1991)

Anderson, E., D. Kern, "Nanofabrication of Zone Plates for X-ray Microscopy," in Xray Microscopy III, A.G. Michette, G.R. Morrison, C.J. Buckley, Eds., Springer Series in Optical Sciences 67 (SpringerVerlag, New York, 1992), 75-78.

Attwood, D.T., "New Opportunities at Xray Wavelengths," Physics Todoy, Am. Inst. Physics, NY (August 1992).

Attwood, D., C. Sommargren, R. Beguiristain, K. Nguyen, J. Bokor, N.Ceglio, K. Jackson, M. Koike, and J. Underwood, "Undulator Radiation for Extreme Ultraviolet Interferometry of Projection Lithography Optics," Appl. Opt. (to he published)

Balhorn, R., M. Corzett, M. Allen, C. Lee, T. Barbee, Jr., J. Kuch, B. MacGowan, D. Matthew's, S. Mrowka, J. tRebes, I. McNulty, L.Da Silva, J. Gray, E. Anderson, D. Kern, D. Attwood, "Application of Xrays to the Analysis of DNA Packaging in Mammalian Sperm," in Soft X-ray Microscopy, C. Jacobsen, J. Trebes, Eds., Proc. SPIE 1741, 43 (1992)

DaSilva, L.B., J.E. Trebes, R. Balhorn, S Mrowka, E. Anderson, D.T. Attwood, T.W. Barbee, Jr., J. Brase, M. Corzett, J. Gray, J.A. Koch, C. Lee, D. Kern, R.A. London, B.J. MacGowan, D.L. Matthews and G. Stone, "'X-ray Laser Microscopy of Rat Sperm Nuclei," Science' 258, 269 (1992).

De Stasio, G., C. Capasso, W. Ng, A.K. Ray-Chaudhuri, S.H. Liang, R.K. Cole, Z.Y. Guo, J. Wallace, F. Cerrina, G.

Margaritondo, J. Underwood, R. Perera, J. Kortright, D. Mercanti, M.T. Ciotti, and A. Stecchi, "High Resolution Photoelectron Microimaging of Neuron Networks," Europhysics Letters, 16, 411 (1991).
Dilmanian, F.A., E. Nachaliel, R.F. Garrett, W.C. Thomlinson, L.D. Chapman, H.R Moulin, T. Oversluizen, H.R. Rarback, M. Rivers, P. Spanne, A.C. Thompson and H.D. Zeman, "Multiple Energy Computed Tomography with Monochromatic X-rays from the NSLS," IEEE Conf. Record of the Medical Imaging Conference, 3, 1831 (1992).

Goncz, K., M. Moronne, W. Lin, S Rothman, "Measuring Changes in the Mass of Single Subcellular Organelles using X-ray Microscopy," in Soft X-ray Microscopy, C. Jacobsen, J. Trebes, Eds., Proc, SPIE 1741, 40 (1992)

Gullikson, E.M., J.H. Underwood, P.C. Batson, and V. Nikitin, "A Soft X-ray/EUV Reflectometer Based on a Laser-I'roduced Plasma Source," I. of X-ray Sti. and Ti'th , 3 , $258(1992)$

Henke, B.L., E..M. Gullikson and J.C. Davis, "X-Ray Interactions: Photuabsorption, Scattering, and Reflection. $\mathrm{E}=50-30,000 \mathrm{eV}, \mathrm{Z}=1.92$," Alomic Data and Nuclear Data Tables , 54 No. 2 (Academic P'ress, San Diego (1993).

Kagoshima, Y., J. Wang, M. Ando, S. Aoki, E. Anderson, D. Attwood, and D. Kern, "70 non-Resolution Zone Plate Soft X-ray Microscope using Undulator Radiation," lapm. J. Appl. Pllysics (in press)

Koch, J.A., B.J. MacGowan, L.B. DaSilva, D.L. Matthews, J.H. Underwood, P.J Batson, and S. Mrowka, "Observation of Gain-Narrowing and Saturation in Se Xray Laser Line Profiles," Phys. Re'v. Lett. 68, $3291(1992)$

Koike, "Beam Splitting of Undulator Radiation with Variable Spacing Plane Grating Monochromator," Nicl. Instru. Methots, A319, 135-140 (1992)

Kortright, J.B., E.M. Gullikson, and P'.E. Denham, "Masked Deposition Techniques for Achieving Multilayer Period Variations Required for Short Wavelength (68A) Soft X-ray Imaging Optics," Applici Optics (accepted).

Kortright, J.B., H. Kimura, V. Nikitin, K. Mayama, M. Yamamoto, and $M$. Yanagihara, "Soft X-ray $(97 \mathrm{eV})$ Phase Retardation Using Transmission Multilayers," Applied Physics Letters, 60 , 2963 (1992)

Matthews, D. L., D. Minyard, G. Stone, T. Yorkey, E.H. Anderson and D.T. Attwood, "Demonstration of X-Ray Microscopy with an $X$-Ray Laser Operating near the Carbon K Edge," Optics Letters, 17, 754 (1992).
Publications 
McNulty, I., J. Kirz, C. Jacobsen, E.H Anderson, M.R. Howells, and D.P. Kern, "High-Resolution Imaging by Fourier. Transform X-Ray Holography," Science, 256, $110(19$ (1992).

Meyer-llso, W, P. Ciuttmann, J. Thieme, 1). Rudolph, C. Schmahl, E. Anderson, l'. Batson, D. Attwood, N. Iskinder, D. Kern, "Experimental Characterization of Zone Plates for High Resolution X-ray

Microscopy," in X-rony Mierosicony III, A.Ci. Michette, G.R. Morrison, C.J. Buckley, Ed ., Springer Series in Optical Sciences 67 (s minger-Verlag, New York, 1942), 284. $2 x, 4$.

Mever-llse, W., M. Koike, H. Beguiristain, J. Maser, 1). Attwood, "X-riny Microscony Resource Center at the Advanced Light Source", in Soff X-riny Microscopy, $C$. Jacobsen, J. Trebes, Eds., I'roc. Sl'IE 1741, 4 (1992).

Mever-Hse, W., D. Attwoud, and M. Kuike "X-ray Micioscopy Resource Center at the Advanced light Source," in Procesedings of the BSR '42 Conference on Blolengy and Synchrotron Rodiation: Symlerotron

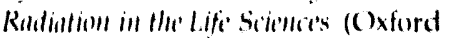
University l'ress, New York, 164.3).

T. Namionks and M. Kuike, "Anslytical Representation of Spot Diagrams and its Applicintion to the Design of Monochrometers," Ninl. Inst. and Mteth. A319, 219.227 (1992)

T. Namioka and M. Koike, "Design of Compact High-Resolution For-Ultraviolet spectrographe Eepuipped with a Cirating Hawing Variable Spacing Curved Ciroovess," LV and X-Rayl Siptetroscoply of

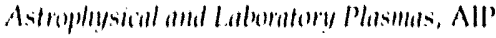
conference l'roceedings (in press).

Nechalied, R., F.A. Dilm,anian, R.I. Cinredt, W.C. Thomlinson, N.Ci. Gmur, N.M. L.azarr., H.R. Moulin, M.L.. Rivers, H. Rarback, PיM. Stefon, I'. Spanne, I',N. l.uke, R. I'ehl, A.C. Thompsom and M. Miller, "Monochromatic Compuled Tomography of the Homon Broin using Synchroten X-Roys: Technical Fensibility,"

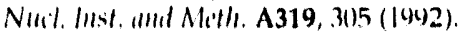

Nguyem, K.B., T. D. Nguyen, D.T. Athwond, T.K. Ciustafson, "Step Covernge I'rofile and l'ropagation of Roughness of Sputter-beposited Multiayer Couting for IUV Projection l.ithography,"l. Viri, sii. Trithmol. (to be published).
Nguyen, K.B., T. D. Nguyen, A.K. Wong, A.R. Neureuther, D.T. Attwoed, "Areal Imoges of EUV I'rojection Lithegraphy Masks with Defects in Reflective Coatings: Electromagnetic Stimulation," OSA Proverdings for soft X-roly I'rojertion litheserifluy' 9.3 (to bo published).

Nguyen, T.D., R. Gronsky, and J.B. Kortright, "Microstructural Evolution of Nanometer Ruthenium Cilms Between Carbon Layers with Thermal Treatments," Mut. Re's. Sict. Proci, 230, 109(1942)

Smith, H.I., S.D. Hector, M.L., Schatternburg, and E.H. Andersen, "A Now Approach fo High Fidelity E. Beam and Ion-Beam Lithography Based on an In Situ

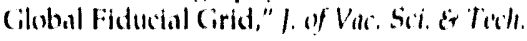
$B, 6,2942$ (1942)

Thumlinson, W., N. (imur, D). (hopmon, R. Cisrett, N. Lazars, H. Moulin, A.C. Thompson, H.D. Zemon, Cis. Brown, J. Murrison, l', Reiser, V. l'admanabalun, L. Ong, S. Cireen, J. Cilacomini, H. Ciordon and E. Rubenstein, "First (operation of the Medical Research Ficility at the NSLS for Coronury Angiography," Re't. Sii. Instr. 63, 625 (1492).

Thumpson, A.C., K.L. Chapman it il., "Focussing (Optics for a Synchrotrom-Based X-Royl Micreprobe, "Neril. Imst. Imtil Me'th. A319, 3015 (1042)

Thompson, A.C., K.L., Chapm,an, and J.H. Underwered, "Developement of an X-ray Microprobe Using Synchrotron Radiation," sille Proc of Cont onc optics for HighBrighthe'ss Synchrotron Beamlines, July I442 (in press).

Underwoud, J.H., E.M. Gullikson, and K. Nguyen, "Tornishing of Mo/si Multilayer $X$-ray Mirrors," Applied (eptics (to be published).

Underwoud, I.H., I.B. Kortright, R.C.C. Rerera, F. Corrina, C. Capalsos, A.K. RayChatudhuri, W. Ng, J. Welnak, J. Wallace, S. liang, and C. Margaritundo, "X-ray Microscopy with Multilayer Mirrors: the MAXIMUM I'hutoelectron Microscope," Procedings of the BSR '92 Conference:

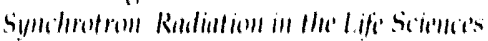
(C)ford University P'ress, New York. $199,3)$.

Yen, A., E.H, Anderson, R.A. Cilunbari, M.L. Sihnttenburg, and H.I. Simith, "Achromatic Holographic Configuration for I 10(1)-nm-Period l ithography," Applad ()plicis, 31, 45+1) (1902) 

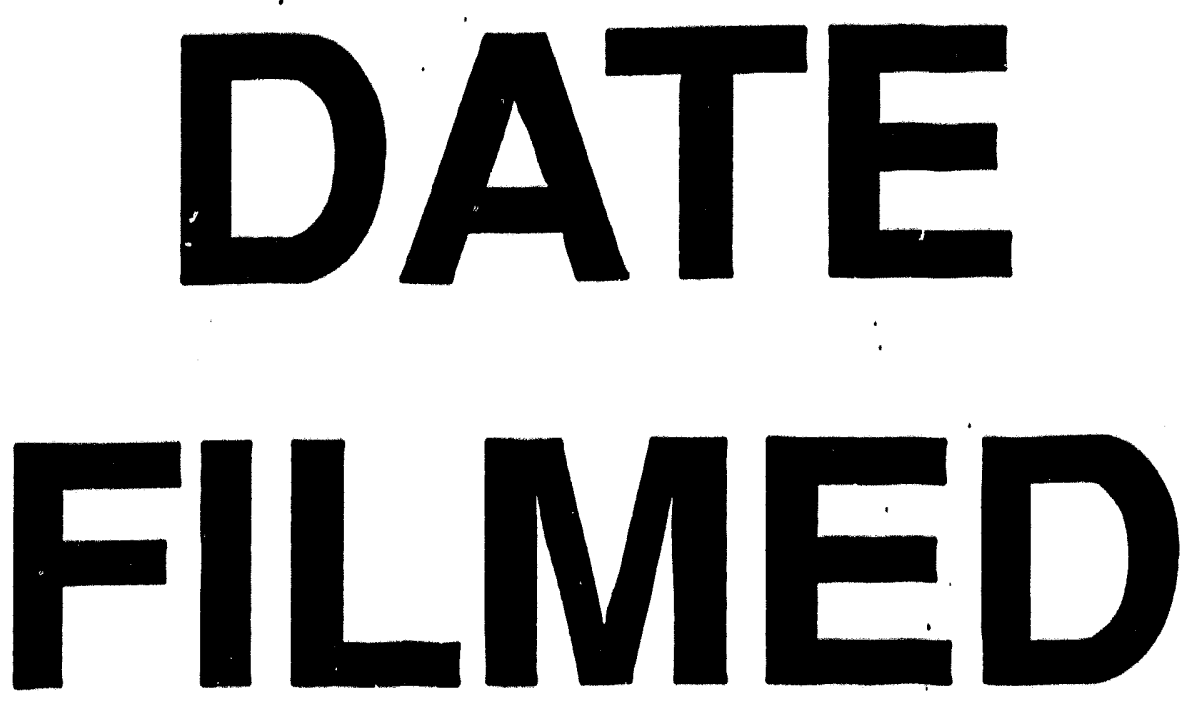

$11 / 9 / 93$
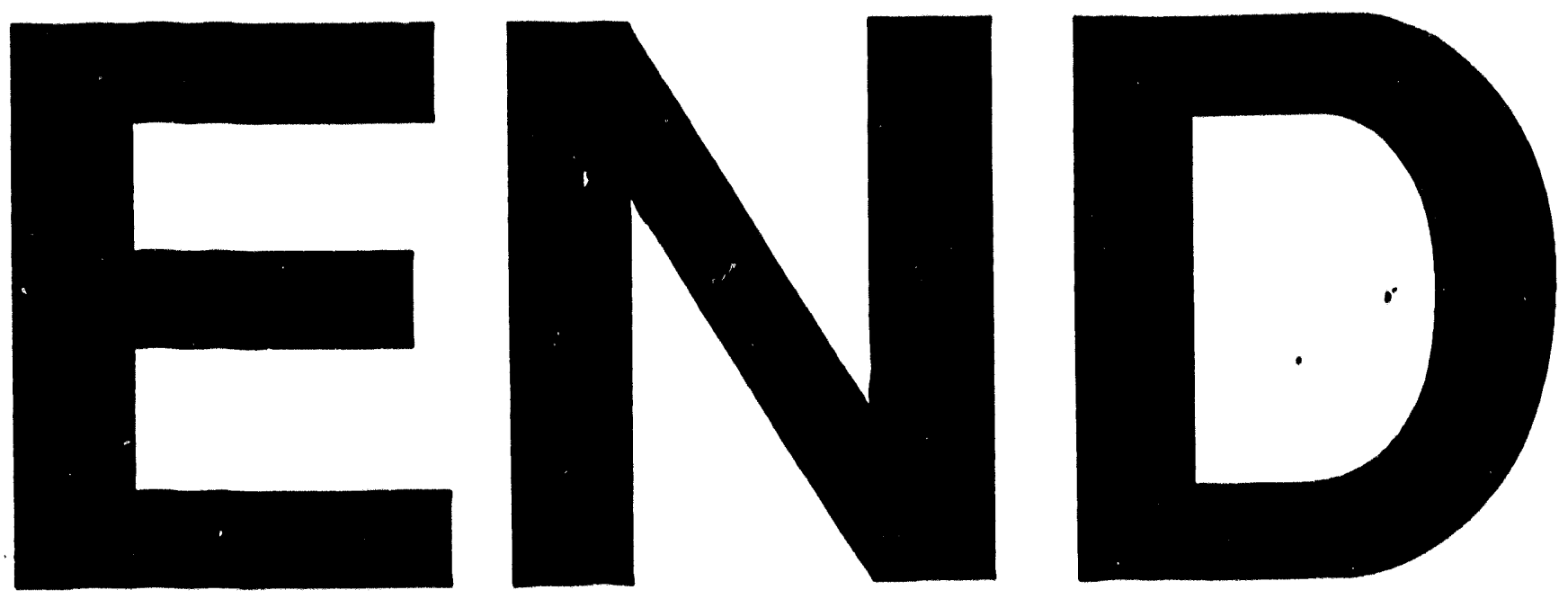
